\title{
Long Non-Coding RNA HCP5 Functions as A Sponger of miR-29b-3p and Promotes Cell Growth and Metastasis in Hepatocellular Carcinoma Through Upregulating DNMT3A
}

\author{
Yongping Zhou \\ Wuxi Second Hospital \\ Kuan Li \\ Kunshan Hospital of Traditional Chinese Medicine \\ Tu Dai \\ Wuxi Second Hospital \\ Hong Wang \\ Wuxi Second Hospital \\ Zhiyuan Hua \\ Wuxi Second Hospital \\ Wuyang Bian \\ Wuxi Second Hospital
}

Hao Wang

Wuxi Hand Surgery Hospital

Fangming Chen

Wuxi Second Hospital

Xiaoming Ai ( $\nabla$ aixiaoming0503@tom.com )

The Affiliated BenQ Hospital of Nanjing Medical University

\section{Research}

Keywords: HCP5, hepatocellular carcinoma, miR-29b-3p, DNMT3A

Posted Date: June 23rd, 2020

DOI: https://doi.org/10.21203/rs.3.rs-37467/v1

License: () (1) This work is licensed under a Creative Commons Attribution 4.0 International License. Read Full License 


\section{Abstract}

Background: Multiple researches have revealed that long non-coding RNA (IncRNAs) served as regulatory factor in modulating tumorigenesis of hepatocellular carcinoma (HCC). In this study, we demonstrated that IncRNA HCP5 was a tumor promoting factor of HCC, and the molecular mechanism of HCP5 has been elucidated at length.

Methods: BrdU, transwell assay and flow cytometry were exerted to estimate cell growth, metastasis, invasion and apoptosis of HCC in vitro. Animal studies were performed the effect of HCP5 in vivo. Furthermore, we identified that miR-29b-3p interacted with HCP5 or DNA methyltransferase 3A (DNMT3A) using dual luciferase assay. qRT-PCR, western blot, BrdU, tanswell and flow cytometry assays were used to investigate the correlation of HCP5/miR-29b-3p/DNMT3A axis.

Results: In present research, we elaborated that HCP5 was overexpressed in HCC tissues and cell lines, and this phenomenon was even obvious in metastatic and recurrent cases. Knockdown of HCP5 significantly alleviated cell growth, metastasis and invasion through preventing apoptosis and activating EMT progress. Moreover, miR-29b-3p has been identified as a negatively regulatory target gene of HCP5, which served as tumor suppressor of HCC to prevent cell proliferation, migration and invasion. Subsequently, DNMT3A was found as a downstream regulatory factor of miR-29b-3p, of which study found that a participated element of HCC progression by activating AKT phosphorylation.

Conclusion: Generally, our study elucidated for the first time that HCP5 plays a crucial role in HCC via HCP5/ miR-29b-3p/DNMT3A/AKT axis and demonstrated a novel diagnostic and therapeutic strategy with potentiality.

\section{Introduction}

Liver cancer is a common digestive malignant cancer in the world, and hepatocellular carcinoma is the predominant histological type of liver cancer. Hepatocellular carcinoma has been ranked as the third leading cause of cancer mortality with high incidence rate ${ }^{1}$. It's reported that roughly seven hundred thousand people die of hepatocellular carcinoma (HCC) worldwide annually ${ }^{2}$. Although there are multiple treatment methods used in curing HCC, its poor prognosis and high degree of malignancy are still hindrances in improving HCC patients lifespan ${ }^{3}$. Additionally, due to the deficiency of early symptoms and specific biomarkers, a majority of HCC patients are already at the advanced phase or develop metastasis when being diagnosed ${ }^{4}$. Generally, the migration to peripheral or distant tissues is recognized as the pivotal factor for recurrence of $\mathrm{HCC}^{5}$. Because of its aggressiveness and easy recurrence, traditional treatment methods, such as surgical resection, liver transplantation, radiotherapy, and chemotherapy, lack efficient treatment effect. Therefore, investigation on biomarkers for early diagnosis and therapy strategies has become the research focus in hepatocellular carcinoma fields. 
Recently, many researches discovered that there are some pathological correlation between long noncoding RNA (IncRNAs) and various malignant carcinomas, including gastric cancer, breast cancer, colorectal cancer and non-small cell lung cancer ${ }^{6-10}$, which make IncRNAs a hotspot of cancer therapy fields. Accumulating evidence elucidated that some IncRNAs function as sponge of microRNAs (miRNAs) and therefore mediating tumorigenesis process of hepatocellular carcinoma. Research performed by $\mathrm{He}$ et al. illustrated that IncRNA maternally expressed gene-3 (MEG-3) prevented cell proliferation, metastatic and invasive capacity via negatively regulating miR-664 ${ }^{11}$. Moreover, Li et al. demonstrated that metastasis-associated lung adenocarcinoma transcript 1 (MALAT-1) also functioned as molecular sponge of miR-146b-5p and therefore promoting cancer cell growth and invasion of $\mathrm{HCC}^{12}$.

Human histocompatibility leukocyte antigen (HLA), HLA complex P5 (HCP5) has been recognized as a key factor in immune system cells with potential regulatory effects in autoimmunity ${ }^{13}$. So far, many studies have proposed the pathological association between HCP5 and various cancers. It's elaborated that HCP5 could promote colon cancer via activating AP1G1 through PI3K/AKT pathway, and exacerbate triple negative breast cancer via acting as a ceRNA to modulate BIRC3 and down-regulate miR-219a-5 $p^{14}$, 15. Additionally, knockdown of HCP5 could exert tumor-prevented effect by up-regulating miR-128-3p in anaplastic thyroid cancer ${ }^{16}$. Although HCP5 was reported as a susceptibility locus for HCV-related HCC through the meta-analyses conducted by Christian et al. ${ }^{17}$, the exact function of HCP5 in HCC still remains further elucidation.

In recent decades, miRNAs have been revealed as tumor regulatory factors, which participate in many process including cell cycle, apoptosis and epithelial-stromal transformation ${ }^{18,19}$. Jinmal el at. indicated that abbreviated miRNAs expression are pathologically correlated to HCC with hepatitis infection, cirrhosis, and patient survival ${ }^{20}$, which indirectly suggested the participation of miRNAs in HCC. Recently, miR-29b-3p was proved as the tumor promoting factor of bladder cancer via suppressing DNA methyltransferase 3A (DNMT3A) ${ }^{21}$. Although the abbreviation of miR-29b-3p was observed in many kind of cancers including bladder cancer, colorectal cancer, and pancreatic cancer ${ }^{22,23}$. There is still no evidence about the specific function of miR-29b-3p in hepatocellular carcinoma, which encourages us to lucubrate the related signal pathway in HCC.

\section{Materials And Methods}

\section{Patients' samples and cell culture}

Hepatocellular carcinoma tissues and adjacent normal tissues were resected through surgical operation from HCC patients in Wuxi Second Hospital and The Affiliated BenQ Hospital of Nanjing Medical University. The extracted tissues were immediately frozen in liquid nitrogen. All human participants related experiments were conducted in agreement with the ethical standards of the ethics committee of Wuxi Second Hospital and The Affiliated BenQ Hospital of Nanjing Medical University as well as the 1964 Helsinki Declaration. All involved patients have sighed the informed consent documents. Subsequently, 
patients' clinicopathological and demographic data was gleaned and described in Table 1. Human hepatic cell line (LO2) and human hepatocellular carcinoma cell lines (Hep3B, HCCLM3, Huh7, MHCC-97H and SMMC-7721) were cultured using DMEM with $10 \% \mathrm{FBS}$ at $37^{\circ} \mathrm{C}, 5 \% \mathrm{CO}_{2}$. 
Table 1 Association analysis of IncHCP5 expression and the clinicopathological features in $80 \mathrm{HCC}$ patients. The median expression level was used as the cut-off.

\begin{tabular}{|c|c|c|c|c|}
\hline \multirow[t]{3}{*}{ Characteristics } & \multirow[t]{3}{*}{ Case number } & \multicolumn{2}{|c|}{ IncHCP5 } & \multirow[t]{3}{*}{$P$-value } \\
\hline & & Low & High & \\
\hline & & $(n=40)$ & $(n=40)$ & \\
\hline Number & 80 & 40 & 40 & \\
\hline Ages (years) & & & & 0.4957 \\
\hline$<65$ years & 47 & 25 & 22 & \\
\hline$\geq 65$ years & 33 & 15 & 18 & \\
\hline Gender & & & & 0.1990 \\
\hline Male & 61 & 33 & 28 & \\
\hline Female & 19 & 7 & 12 & \\
\hline AFP, $\mu \mathrm{g} / \mathrm{L}$ & & & & 0.4123 \\
\hline$<400 \mathrm{ng} / \mathrm{ml}$ & 17 & 7 & 10 & \\
\hline$\geq 400 \mathrm{ng} / \mathrm{ml}$ & 63 & 33 & 30 & \\
\hline Cirrhosis & & & & 0.5762 \\
\hline Absent & 16 & 9 & 7 & \\
\hline Present & 64 & 31 & 33 & \\
\hline Tumor size & & & & 0.0008 \\
\hline$\leq 5 \mathrm{~cm}$ & 32 & 23 & 9 & \\
\hline$>5 \mathrm{~cm}$ & 48 & 17 & 33 & \\
\hline Tumor number & & & & 0.1305 \\
\hline Single & 46 & 27 & 22 & \\
\hline Multiple & 34 & 13 & 21 & \\
\hline Vascular invasion & & & & 0.0013 \\
\hline Absent & 18 & 3 & 15 & \\
\hline Present & 62 & 37 & 25 & \\
\hline TNM stage & & & & 0.0116 \\
\hline | - || & 49 & 30 & 19 & \\
\hline
\end{tabular}




\begin{tabular}{|lcccc|}
\hline III-IV & 31 & 10 & 21 & \\
Edmondson & & & & 0.0005 \\
I- II & 51 & 33 & 18 & \\
III + IV & 29 & 7 & 22 & \\
Capsular & & & & \\
Present & 53 & 25 & 28 & 0.4781 \\
Absent & 27 & 15 & 12 & \\
\hline
\end{tabular}

a Two-sided chi-squared test.

\section{Quantitative reverse transcriptase polymerase chain reaction (qRT-PCR)}

Human HCC cell lines (Hep3B, HCCLM3) with different treatments were collected in logarithmic growth period and mixed with $1 \mathrm{~mL}$ of TRIzol. Chloroform were added for 15-min maintenance at room temperature, then the solution was added with isopropyl alcohol and centrifuged to obtain the RNA sediment. Finally, the RNA extraction was dried and hoarded under $-80^{\circ} \mathrm{C}$ till experiments. QRT-PCR analyses of HCP5, miR-29b-3p and DNMT3A were performed using PrimeScript RT reagent Kit and SYBR Prime Script RT-PCR Kits based of the protocol. The transcription level was subsequently analyzed by $2^{-\triangle \Delta C t}$ method. GAPDH was utilized as internal control.

\section{Western blot}

Cells were collected and lysed in lysis buffer on ice for $30 \mathrm{~min}$. Then, the protein quantity was calculated using BCA Kit. Proteins solution was then added with loading buffer and boiled for $5 \mathrm{~min}$. Subsequently, proteins were transferred onto polyvinyldifluoride membranes (Bio-Rad Laboratories USA) after separated on SDS-PAGE. The membranes were immersed with the diligent of HCP5, miR-29b-3p, DNMT3A, AKT, pAKT and GAPDH proteins' antibodies, while GAPDH was used as internal control. Then observation was performed through an enhanced chemiluminescence reagent (Thermo Scientific, Waltham, MA, USA).

\section{Cell transfection}

The specific small RNAs against HCP5, DNMT3A (sh-HCP5, si-DNMT3A) and small RNA negative control (si-NC), were purchased from Sangon Biotech Co., Ltd. (Shanghai, China). Sh-HCP5, sh-Control, siDNMT3A and si-NC were transfected into Hep3B and HCCLM3 cell lines to knockdown HCP5, DNMT3A based on the manufacture's protocol, respectively. MiR-29b-3p mimics and negative control (miR-NC) were used to treat Hep3B and HCCLM3 cell lines.

\section{Dual-luciferase assay}


pGL3 vector (Promega Corporation, Madison, USA) and the synthetic HCP5 containing wild-type (WT) or mutated (Mut) region (Sangon, Shanghai, China) were utilized to construct the reporter plasmids, which were then co-transfected into cells with miR-29b-3p inhibitor using Lipofectamine 2000 under manufacturer's instruction. Also, negative control inhibitor was used to generate the control group. DualLuciferase Reporter Assay System and luminometer were utilized to estimate the Renilla and Firefly luciferase activities after $24 \mathrm{~h}$.

\section{Transwell chamber assay}

Lucubration of the capacity of cell migration, roughly $1 \times 10^{5}$ cells was collected and then transferred onto the upper compartment of a Transwell chamber囚which has an $8 \mu \mathrm{m}$ pore size and a 24-well insert. In the upper chamber of each well, $50 \mu \mathrm{L}$ of serum-free medium containing $10 \mathrm{~g} / \mathrm{L}$ bovine serum albumin was added. All the lower chambers were loaded with $10 \%$ FBS. Cell migration capacity was assessed by the number of cells reaching the lower chamber. For invasion assays, the upper chamber were enfolded with Matrigel (BD Biosciences, San Jose, CA, USA). The other procedures were as the migration assays.

\section{Apoptosis Level evaluated by Flow Cytometry}

Flow cytometry was exerted to study the effects on cell cycle and apoptosis after transfections. Annexin V-FITC Apoptosis Detection Kit and Cell Cycle Detection Kit were used to stain cells. Cells were treated based on the manufacture's protocol.

\section{Cell proliferation determination}

After cell transfection, cells in logarithmic growth phase were then seeded into 96 -wells plate with $1 \times 10^{5}$ cells in each well. Subsequently, BrdU reagent was diluted using DMEM medium and was added to stain cells according to manufacturer's protocol. Gray level images were acquired under a laser scanning microscope (Axioskop 2 plus, Carl Zeiss Co.Ltd., Jena, Germany).

\section{Animal studies}

To further investigate the exact function of HCP5 on BALB/c nude mice with $\mathrm{HCC}$, all nude mice were raised and maintained in a pathogen-free environment at the Nanjing Medical University. All animal experiments were carried out in accordance with the National Institutes of Health guide for the care and use of laboratory animals, and approved by the ethics committee of Nanjing Medical University. The nude mice were then allocated into two group randomly, one group is sh-Control group, and another is sh-HCP5 group. A total of $100 \mu \mathrm{l}$ of HCCLM3 cells (with sh-control or sh-HCP5 transfection) $\left(1 \times 10^{6}\right)$ PBS suspension were injected into the mammary fat pads of nude mice to construct a stable HCC animal model, and tumor were allowed to grow till a diameter of roughly $5 \mathrm{~mm}$. Three weeks after injection, nude mice were executed, and HCC tumor was extracted for observation. Next, the volume of tumor on nude mice were recorded and photographed. The extracted HCC cancer tissues were sliced for further examination. 


\section{Immunohistochemical staining (IHC)}

Dewax, dehydrate and rehydrate the slices. Primary antibody (1:100, cell signal, Danvers, MA, USA) was added to the sections and incubated overnight at $4^{\circ} \mathrm{C}$. Then the biotinylated secondary antibody (Goldenbridge, Zhongshan, China) was applied according to the SP-IHC test.

\section{Statistical analyses}

The results data were expressed as mean plus standard deviation (SD) for triplicate measurements. Statistically significant differences in different groups were evaluated by Student's $t$ test through SPSS (13.0) or Graphpad Prism 7.0. P 0.05 means statistically significance.

\section{Results}

\section{LncRNA HCP5 was in high expression level in HCC tissues and cell lines and was closely correlated with HCC progression}

To examine the HCP5 expression quantities in HCC related tissues and cell lines, qRT-PCR was conducted to determine 80 pairs of stochastically extracted HCC tissues and adjacent normal tissues. In present study, we indicated that HCP5 was overexpressed in HCC tissues versus adjacent normal tissues $(\mathrm{p}<0.001$, Fig.1A). Simultaneously, HCP5 was also examined in higher expression level in HCC cell lines (Hep3B, HCCLM3, SMCC-7721, Huh7 and MHCC-97H) when compared with normal hepatocyte L02 $\left({ }^{* \star *} p<0.001,{ }^{* \star} p<0.01\right.$, respectively, Fig.1B). Thus, these consequences collectively suggested that overexpression of IncRNA HCP5 might be associated with HCC.

Next, we further examined the association between the clinical parameters and HCP5 expression. As shown in Table 1, HCP5 was significantly higher in large tumor size, metastasis, high histological grade tissues and recurrence $\left({ }^{* \star} p<0.01,{ }^{\star \star \star} p<0.001\right.$, Fig. $\left.1 \mathrm{C}\right)$. Generally, these results illustrated that HCP5 probably plays an important role in regulating the progression of HCC.

\section{HCP5 promoted cell growth, metastasis and invasion through prohibiting apoptosis in HCC in vitro and vivo}

Lucubration of the specific function of HCP5 in HCC was performed by transfecting si-HCP5 into Hep3B and HCCLM3 cell lines to knockdown HCP5. Cells have reached ideal transfection states after $24 \mathrm{~h}$. As depicted in Fig.2A, HCP5 was in significantly lower expression in HCC cell lines (Hep3B and HCCLM3) with sh-HCP5 transfection. Subsequently, cell proliferative capacity was attenuated in Hep3B and HCCLM3 with HCP knockdown using BrdU assay $\left({ }^{*} \mathrm{p}<0.05\right.$, ${ }^{*} \mathrm{P}<0.01$, Fig.2B). Moreover, the metastatic and invasive abilities in $\mathrm{HCC}$ cells were investigated by transwell chamber assay. The results demonstrated that HCP5 knockdown in Hep3B and HCCLM3 cell lines resulted in alleviated cell metastatic and invasive quantities ( ${ }^{\star} p<<0.01, \star \star \star P<0.001$, Fig. $2 \mathrm{C}$ and $2 \mathrm{D}$ ), which indicated that the existence of HCP 5 could functionally augment the metastatic and invasive abilities in HCC. 
As we have proved that overexpression of HCP5 enabled the proliferation, metastatic and invasive ability of $\mathrm{HCC}$, the exact modulatory process is still not clear. Therefore, flow cytometry was conducted to further scrutinize whether the alteration of HCP5 expression would thereby affect cell apoptosis. The results of flow cytometry showed that the apoptosis rate was negatively proportional to HCP5 expression level, which meant that down-regulation of HCP5 lead to higher apoptosis level ( $\star \star \star p<0.001$, Fig.2E). Additionally, we have estimated the expression level of epithelial-mesenchymal transition (EMT) related proteins (E-cadherin and Vimentin) by Western blot. As shown in Fig.2f, knockdown of HCP5 resulted in high expression of E-cadherin and down-regulation of Vimentin in HCC cell lines (Hep3B and HCCLM3) (Fig.2F), which illustrated that down-regulation of HCP5 might promote HCC cell metastasis and invasion via activating EMT process.

Although we have found the evidences that HCP5 could promote HCC proliferation, metastasis and invasion in vitro, the in vivo effect of HCP5 on HCC progression still need to be proved. Consequently, we injected HCCLM3 cells into nude mice to construct HCC animal models. The tumor volume was recorded every 3 days after xenograft. The tumor volume of si-HCP 5 transfected group escalated lower than control group (Fig.3A), which further indicated that down-regulation of HCP5 could slow HCC progression. Then tumor in nude mice was extracted and made into slices. HE staining was used to scrutinize the nuance between overexpression and down-regulation of HCP5. Consistent with the in vitro consequence, knockdown of HCP5 destroyed the HCC tumor tissues (Fig.3B). Additionally, the metastasis phenotype was also examined and the results shown in Fig.3C suggested that knockdown of HCP5 led to higher Ecadherin expression and lower Vimentin expression when compared with sh-NC transfected group $(\mathrm{p}<0.05)$. Moreover, Ki67 was utilized to estimate the HCC proliferation in vivo. Consistent to our hypothesis, down-regulation of HCP5 significantly reduced Ki67 positive staining cells (Fig.3D), which demonstrated that HCP5 is a pivotal factor in promoting HCC cell growth.

\section{miR-29b-3p interacted with HCP5}

To further explore the downstream regulatory molecular mechanism of HCP5, we exerted starBase v3.0, miRanda and LncBase to find potential target gene of HCP5. The intersection of prediction targets of the three databases showed that there was a covalent binding fragment of HCP5 wild type binding sites on the miR-29b-3p gene sequence (Fig.4A), which encouraged us to further investigate the exact correlation between miR-29b-3p and HCP5 in HCC. Dual luciferase assay demonstrated that the significant reduction in luciferase activity between wtHCP5 and miR-29b-3p, which explained that miR-29b-3p was negatively regulated by HCP5 in HCC ( ${ }^{\star *} p<0.01$, Fig.4B). The expression level of miR-29b-3p in HCC tissues and adjacent normal tissues were also determined. As expected, miR-29b-3p was expressed in a low quantities level in HCC tissues when compared to normal tissues ( ${ }^{\star *} p<0.01$, Fig.4C), from which we speculated that miR-29b-3p might serve as a tumor suppressor in HCC. Also, the specific expression level between miR-29b-3p and HCP5 was analyzed through qRT-PCR and the result showed that miR-29b-3p and HCP5 is negatively correlated (Fig.4D and 4E). The result depicted in Fig.4D elucidated that knockdown of HCP5 could increase miR-29b-3p expression ( $\left.{ }^{\star \star *} p<0.001\right)$. When given miR-29b-3p mimic, 
HCP5 was shown in low expression level. While given miR-29b-3p, HCP5 was found down-regulation $(p<0.05$, Fig.4E), which suggested that miR-29b-3p and HCP5 were in negative feedback regulation cycle.

\section{miR-29b-3p prevents HCC cell progression brought by HCP5}

To explore the exact function of miR-29b-3p in HCC, miR-29b-3p mimic and miR- NC were used to treat HCCLM3 cells. qRT-PCR was conducted to estimate the miR-29b-3p expression level after the treatment of above reagent. MiR-29b-3p was in high expression level when giving miR-29b-3p mimic to HCC cell lines (HCCLM3 and Hep3B) compared to miR-NC group ( $* \star * p<0.001$, Fig.5A). BrdU assay demonstrated that high expression level of miR-29b-3p resulted in lower proliferative rate $\left({ }^{*} p<0.05,{ }^{*} p<0.01\right.$ compared to miR-NC, Fig.5B), which directly illustrated that miR-29b-3p has the ability to prevent HCC cell proliferation. Additionally, cell metastatic and invasive capacity have been alleviated when HCC cell lines (HCCLM3 and Hep3B) were given miR-29b-3p mimic ( ${ }^{\star \star} p<0.01, \star \star \star p<0.001$, Fig. $5 \mathrm{C}$ and $5 \mathrm{D}$ ). Those results collectively elucidated that miR-29b-3p is capable to inhibit HCC cell proliferation, metastasis and invasion, and thereby prevent the exacerbation of HCC. Next, flow cytometry was conducted to examine the effect of miR-29b-3p on cell apoptosis, and the consequence demonstrated that the apoptosis rate was higher in miR-29b-3p mimic group ( $* \star \star p<0.001$ compared with miR-NC, Fig.5E), which suggested that miR-29b-3p prevent cell growth through improving cell apoptosis. Also, western blot demonstrated that high quantities of miR-29b-3p led to up-regulation of E-cadherin and down-regulation of Vimentin (Fig.5F).

To investigate the correlation between miR-29b-3p and HCP5, miR-29b-3p antagomir was given to shHCP5 transfected HCCLM3 cell line. qRT-PCR showed that knockdown of HCP5 has higher miR-29b-3p quantities, while giving miR-29b-3p antagomir, the expression of miR-29b-3p increased ( $* \star \star p<0.001$, Fig.6A). Additionally, the proliferation of miR-29b-3p antagomir treated HCCLM3 with sh-HCP5 transfection was determined using BrdU assay, the result showed that when given miR-29b-3p antagomir, the proliferative rate significantly increased ( ${ }^{*} \mathrm{p}<0.01$, Fig.6B), which indicated that miR-29b-3p was a tumor suppressor of HCC. Moreover, transwell assay demonstrated that miR-29b-3p antagomir further promoted the metastatic and invasive capacity attenuated by sh-HCP5 ( $<<0.05$, Fig.6C-D). Further certification of the mechanism was investigated through flow cytometry and western blot. The research consequences showed that miR-29b-3p antagomir precluded cell apoptosis ( ${ }^{* \star} p<0.001$, Fig.6E), which illustrated that miR-29b-3p antagomir promoted cell growth through preventing apoptosis. Western blot was conducted to scrutinize the expression of EMT correlated proteins (E-cadherin and Vimentin), the results showed that when given miR-29b-3p antagomir, the quantities of Vimentin was increased while Ecadherin was decreased (Fig.6F). Those consequences collectively elucidated that miR-29b-3p precluded cell growth, metastatic and invasive capacity via promoting apoptosis, up-regulating E-cadherin and down-regulated Vimentin.

\section{DNMT3A was proved as a target gene of miR-29b-3p}

Further exploration of the mechanism by which miR-29b-3p modulates the progression of HCC, Targetscan and starBase v3.0 were used to search the direct target gene of miR-29b-3p. Subsequently, 
DNMT3A was found as the downstream target of miR-29b-3p. As shown in Fig.7A, the bioinformatic prediction demonstrated that DNMT3A was indeed a potential target gene of miR-29b-3p. Additionally, dual luciferase assay further certified that there was a direct interaction between miR-29b-3p and DNMT3A ( ${ }^{* \star} \mathrm{p}<0.001$, Fig.7B). Western blot and qRT-PCR were exerted that expression level of DNMT3A when treated miR-29b-3p mimic, the results showed that high quantities of miR-29b-3p would inhibit the expression of DNMT3A ( $\star \star \star p p<0.001$, Fig7C-D). High quantities of miR-29b-3p led to lower expression quantities of DNMT3A, while down-regulation of miR-29b-3p resulted in high expression quantities of DNMT3A ( ${ }^{\star \star *} p<0.001$, Fig.7E), which indirectly elucidated that the expression of DNMT3A was in inverse proportion to miR-29b-3p. Moreover, western blot demonstrated that down-regulated of miR-29b-3p resulted in higher quantities of DNMT3A both in HCCLM3 and Hep3B, while low expression of miR-29b-3p led to up-regulation of DNMT3A, which indicated that the expression of DNMT3A was proportional to HCP5 (Fig.7F). Furthermore, qRT-PCR and western blot showed that knockdown of HCP5 resulted in lower expression quantities of DNMT3A at mRNA and protein levels when compared with sh-control both in Hep3B and HCCLM3 cell lines (Fig.7G-H). Next, the expression quantities of DNMT3A in HCC tissues were determined through western blot, the research consequences demonstrated DNMT3A was up regulated in HCC tissues when compared to adjacent normal tissues (Fig.7I). The results mentioned above collectively illustrated DNMT3A is a crucial participating factor in modulating HCC progression.

\section{Overexpression of DNMT3A promotes HCC cell growth, metastasis and invasion}

Previous studies have proved that DNMT3A played a role in HCC, therefore, we then proposed an assumption that up-regulation of DNMT3A might alter the HCC progression inhibited by miR-29b-3p. Consequently, EV-DNMT3A and EV-control (EV) were transfected into HCC cell lines (HCCLM3 and Hep3B) to up-regulated the expression of DNMT3A. Also, miR-29b-3p mimics and sh-HCP5 transfection were used to determine the correlation of miR-29b-3p, HCP5 and DNMT3A. As shown in Fig.8A, qRT-PCR demonstrated that DNMT3A was down regulated when there was high expression level of miR-29b-3p, while its expression quantities were positively proportional to HCP5 $\left({ }^{*} p<0.05\right)$. Consistent with this result, western blot showed that high-regulation of DNMT3A reversed the effect brought by sh-HCP5 and miR29b-3p mimics (Fig.8B). Subsequently, BrdU assay was exerted to determine the effect of DNMT3A on HCC proliferation, the result showed that overexpression of DNMT3A led to higher cell growth $\left({ }^{*} \mathrm{p}<0.05\right.$, Fig.8C). Next, cell metastatic and invasive ability were examined through transwell chamber assay, the research consequences suggested that both metastatic and invasive capacity had been increased when giving EV-DNMT3A ( ${ }^{*} p<0.05$, Fig.8D-E). Further exploration of the effect of DNMT3A on regulating proliferation, invasion and migration was performed by flow cytometry and examination of the expression level of EMT correlated proteins (E-cadherin and Vimentin). Flow cytometry showed that DNMT3A promoted cell growth through prohibiting cell apoptosis ( ${ }^{*} \mathrm{p}<0.05$, Fig.8F). Western blot showed that high regulation of DNMT3A could up-regulated Vimentin and decreased the expression of E-cadherin (Fig.8g). Those consequences elucidated that the proliferation, metastatic and invasive capacity of HCC was augmented when there were high expression quantities of DNMT3A (Fig.8b-d), and the mechanism of which was prevention of apoptosis and acceleration of EMT process. 


\section{HCP5/miR-29b-3p/DNMT3A axis augments HCC progression via activating AKT phosphorylation}

Current researches have elucidated that DNMT3A plays a pivotal role in modulating tumorigenesis through activating PI3K/AKT pathway in liver cancer and lung cancer ${ }^{24,25}$. To certify the modulatory function of AKT phosphorylation in HCP5/ miR-29b-3p/DNMT3A, we first confirmed that knockdown of DNMT3A significantly decreased AKT phosphorylation by western blot (Fig.9a), the phosphorylation level of AKT was proportional to DNMT3A. Then, IGF-1, the AKT activator was used to treat HCC cell lines with si-DNMT3A transfection, the proliferation was determined through BrdU assay and the result demonstrated that activation of AKT led to enhanced cell growth ( ${ }^{*} \mathrm{p}<0.01$, Fig. 9B). Transwell chamber assay was exerted to scrutinize the effect of AKT on regulating HCC metastatic and invasive capacity. The research consequences demonstrated that AKT activation augmented that the metastatic and invasive capacity of HCC ( ${ }^{\star \star *} p<0.001,{ }^{\star *} \mathrm{p}<0.01$, Fig.9C-D). Further investigation found that inhibition of AKT phosphorylation led to higher cell apoptosis and weaken EMT process ( ${ }^{\star \star *} \mathrm{p}<0.001$, Fig.9E-F), which proved that AKT phosphorylation was important in regulating HCC progression.

\section{Discussion}

In recent decades, non-coding RNAs, which long non-coding RNAs (IncRNAs) and microRNAs (miRNAs) are involved, have gradually become the research focus in cancer therapy fields ${ }^{26,27}$, since accumulating evidences proved that IncRNAs and miRNAs are used as diagnostic markers and novel therapeutic strategies for various cancers ${ }^{28,29}$. In this study, we demonstrated for the first time that IncRNA HCP5 was overexpressed in HCC tissues and cell lines, and its exact function in regulating HCC. Additionally, the expression quantities of HCP5 was pathologically associated with related clinical parameters, including tumor size, metastasis and TNM stage of HCC. These research findings collectively suggested that HCP5 probably plays a crucial role in HCC.

LncRNA HCP 5 has been reported as a promoting factor in tumorigenesis of breast cancer, colon cancer and hepatocellular carcinoma ${ }^{14,15,17}$. In this research, we elucidated that HCP5 promoted HCC progression (proliferation, metastasis and invasion) via inhibiting apoptosis and activating EMT process in vitro and vivo. Previous studies have reported that IncRNAs could serve as ceRNAs for miRNAs and thereby modulate tumor progression ${ }^{30-32}$. Here, we identified miR-29b-3p as the direct target gene of HCP5 through starBase v3.0 and Targetscan database. Dual luciferase assay further confirmed the correlation of miR-29b-3p and HCP5. Also, miR-29b-3p was found as a tumor suppressor and negatively regulatory factor of HCP5 in HCC. Additionally, Edu assay, transwell assay, qRT-PCR, western blot and flow cytometry collectively illustrated that HCP5 promoted HCC progression via repressing miR-29b-3p. To verify whether HCP5 could be used as biomarkers for diagnosis and the prediction of prognosis, we then found evidence that high quantities of HCP5 was significantly correlated with poor clinical features. These results indicated that HCP5 and miR-29b-3p are probably potential diagnostic biomarkers for HCC.

DNA methyltransferase 3A (DNMT3A) was nowadays recognized as a promoting factor in various cancer $^{33}$. In this study, we identified for the first time that DNMT3A participated in promoting the 
progression of HCC. Using starBase v3.0 and Targetscan $\rrbracket$ we found that DNMT3A was a direct target gene of miR-29b-3p, and dual luciferase assay further confirmed this consequence. Furthermore, DNMT3A was inversely associated with the expressions of miR-29b-3p in HCC cell line. Down-regulation of DNMT3A reversed the effects of HCP5 and miR-29b-3p on HCC. Currently, many studies have declared that AKT phosphorylation is regulated by DNMT3A in various carcinomas including pancreatic cancer and lung cancer ${ }^{34-36}$. In present study, we further proved that HCP5 promoted cell growth, metastasis and invasion via miR-29b-3p/DNMT3A/AKT axis in HCC.

\section{Conclusions}

In conclusion, our findings suggested that HCP5 was overexpressed in HCC, and was capable of accelerating cell proliferation, metastatic and invasive capacity via preventing apoptosis and promoting EMT process, through HCP5/miR-29b-3p/DNMT3A/AKT axis. This discovery might provide novel strategy for early diagnosis and better prognostic methods for HCC.

\section{Declarations}

\section{Funding}

This study was supported by grant from Health Research Projects of Jiangsu Provincial Health Committee (H2019045).

\section{Availability of data and materials}

All data generated or analyzed during this study are included either in this article.

\section{Authors' contributions}

Yongping Zhou and Kuan Li devised the research; Tu Dai and Hong Wang gleaned information; Zhiyuan Hua, Wuyang Bian, and Hao Wang conducted the statistical analysis; Yongping Zhou and Kuan Li drafted the manuscript; Fangming Chen and Xiaoming Ai revised and proofread the manuscript. All authors had perused and reached a consensus on the final manuscript.

\section{Ethics approval and consent to participate}

All procedures performed in studies involving human participants were in accordance with the ethical standards of the Research Ethics Committee of Wuxi Second Hospital and The Affiliated BenQ Hospital of Nanjing Medical University and with the 1964 Helsinki declaration and its later amendments. All written informed consent to participate in the study was obtained from HCC patients for samples to be collected from them.

\section{Consent for publication}


Not applicable.

\section{Competing interests}

Declared none.

\section{References}

1. Bray, F.; Ferlay, J.; Soerjomataram, I.; Siegel, R. L.; Torre, L. A.; Jemal, A., Global cancer statistics 2018: GLOBOCAN estimates of incidence and mortality worldwide for 36 cancers in 185 countries. $C A$ Cancer J Clin 2018,68 (6), 394-424.

2. Ferlay, J.; Shin, H. R.; Bray, F.; Forman, D.; Mathers, C.; Parkin, D. M., Estimates of worldwide burden of cancer in 2008: GLOBOCAN 2008. Int J Cancer 2010,127 (12), 2893-917.

3. Attwa, M. H.; El-Etreby, S. A., Guide for diagnosis and treatment of hepatocellular carcinoma. World J Hepatol 2015,7 (12), 1632-51.

4. Chen, J. G.; Zhang, S. W., Liver cancer epidemic in China: past, present and future. Semin Cancer Biol 2011,21 (1), 59-69.

5. Lindsay, C. K.; Sinha, C. C.; Thorgeirsson, U. P., Morphological study of vascular dissemination in a metastatic hepatocellular carcinoma model in the monkey. Hepatology 1997,26 (5), 1209-15.

6. Abbastabar, M.; Sarfi, M.; Golestani, A.; Khalili, E., IncRNA involvement in hepatocellular carcinoma metastasis and prognosis. EXCLI J 2018,17, 900-913.

7. Hao, N. B.; He, Y. F.; Li, X. Q.; Wang, K.; Wang, R. L., The role of miRNA and IncRNA in gastric cancer. Oncotarget 2017,8 (46), 81572-81582.

8. Shen, Y.; Peng, X.; Shen, C., Identification and validation of immune-related IncRNA prognostic signature for breast cancer. Genomics 2020,112 (3), 2640-2646.

9. Bermudez, M.; Aguilar-Medina, M.; Lizarraga-Verdugo, E.; Avendano-Felix, M.; Silva-Benitez, E.; LopezCamarillo, C.; Ramos-Payan, R., LncRNAs as Regulators of Autophagy and Drug Resistance in Colorectal Cancer. Front Oncol 2019,9, 1008.

10. Ji, D.; Wang, Y.; Li, H.; Sun, B.; Luo, X., Long non-coding RNA LINC00461/miR-149-5p/LRIG2 axis regulates hepatocellular carcinoma progression. Biochem Biophys Res Commun 2019,512 (2), 176181.

11. He, J. H.; Han, Z. P.; Liu, J. M.; Zhou, J. B.; Zou, M. X.; Lv, Y. B.; Li, Y. G.; Cao, M. R., Overexpression of Long Non-Coding RNA MEG3 Inhibits Proliferation of Hepatocellular Carcinoma Huh7 Cells via Negative Modulation of miRNA-664. J Cell Biochem 2017,118 (11), 3713-3721.

12. Li, C.; Miao, R.; Liu, S.; Wan, Y.; Zhang, S.; Deng, Y.; Bi, J.; Qu, K.; Zhang, J.; Liu, C., Down-regulation of miR-146b-5p by long noncoding RNA MALAT1 in hepatocellular carcinoma promotes cancer growth and metastasis. Oncotarget 2017,8 (17), 28683-28695.

13. Liu, Y.; Helms, C.; Liao, W.; Zaba, L. C.; Duan, S.; Gardner, J.; Wise, C.; Miner, A.; Malloy, M. J.; Pullinger, C. R.; Kane, J. P.; Saccone, S.; Worthington, J.; Bruce, I.; Kwok, P. Y.; Menter, A.; Krueger, J.; Barton, A.; 
Saccone, N. L.; Bowcock, A. M., A genome-wide association study of psoriasis and psoriatic arthritis identifies new disease loci. PLoS Genet 2008,4 (3), e1000041.

14. Yun, W. K.; Hu, Y. M.; Zhao, C. B.; Yu, D. Y.; Tang, J. B., HCP5 promotes colon cancer development by activating AP1G1 via PI3K/AKT pathway. Eur Rev Med Pharmacol Sci 2019,23 (7), 2786-2793.

15. Wang, L.; Luan, T.; Zhou, S.; Lin, J.; Yang, Y.; Liu, W.; Tong, X.; Jiang, W., LncRNA HCP5 promotes triple negative breast cancer progression as a ceRNA to regulate BIRC3 by sponging miR-219a-5p. Cancer Med 2019,8 (9), 4389-4403.

16. Chen, J.; Zhao, D.; Meng, Q., Knockdown of HCP5 exerts tumor-suppressive functions by upregulating tumor suppressor miR-128-3p in anaplastic thyroid cancer. Biomed Pharmacother $2019,116,108966$.

17. Lange, C. M.; Bibert, S.; Dufour, J. F.; Cellerai, C.; Cerny, A.; Heim, M. H.; Kaiser, L.; Malinverni, R.; Mullhaupt, B.; Negro, F.; Semela, D.; Moradpour, D.; Kutalik, Z.; Bochud, P. Y.; Swiss Hepatitis, C. C. S. G., Comparative genetic analyses point to HCP5 as susceptibility locus for HCV-associated hepatocellular carcinoma. J Hepatol 2013,59 (3), 504-9.

18. He, J. F.; Luo, Y. M.; Wan, X. H.; Jiang, D., Biogenesis of MiRNA-195 and its role in biogenesis, the cell cycle, and apoptosis. J Biochem Mol Toxicol 2011,25 (6), 404-8.

19. Du, R.; Sun, W.; Xia, L.; Zhao, A.; Yu, Y.; Zhao, L.; Wang, H.; Huang, C.; Sun, S., Hypoxia-induced downregulation of microRNA-34a promotes EMT by targeting the Notch signaling pathway in tubular epithelial cells. PLoS One 2012,7(2), e30771.

20. Jiang, J.; Gusev, Y.; Aderca, I.; Mettler, T. A.; Nagorney, D. M.; Brackett, D. J.; Roberts, L. R.; Schmittgen, T. D., Association of MicroRNA expression in hepatocellular carcinomas with hepatitis infection, cirrhosis, and patient survival. Clin Cancer Res 2008,14 (2), 419-27.

21. Yang, L. I.; Liang, Y.; Biology, D. O., MiR-29b-3p promoting the mitomycin resistance of bladder cancer via repressing DNMT3A expression. Acta Academiae Medicinae Wannan 2015.

22. Ding, D.; Li, C.; Zhao, T.; Li, D.; Yang, L.; Zhang, B., LncRNA H19/miR-29b-3p/PGRN Axis Promoted Epithelial-Mesenchymal Transition of Colorectal Cancer Cells by Acting on Wnt Signaling. Mol Cells 2018,41 (5), 423-435.

23. Sun, Y.; Wang, P.; Yang, W.; Shan, Y.; Zhang, Q.; Wu, H., The role of IncRNA MSC-AS1/miR-29b-3p axismediated CDK14 modulation in pancreatic cancer proliferation and Gemcitabine-induced apoptosis. Cancer Biol Ther 2019,20 (6), 729-739.

24. Chen, Q.; Gao, Y.; Yu, Q.; Tang, F.; Zhao, P. W.; Luo, S. K.; Lin, J. S.; Mei, H., miR-30a-3p inhibits the proliferation of liver cancer cells by targeting DNMT3a through the PI3K/AKT signaling pathway. Oncol Lett 2020,19 (1), 606-614.

25. Liu, D.; Wong, C. C.; Fu, L.; Chen, H.; Zhao, L.; Li, C.; Zhou, Y.; Zhang, Y.; Xu, W.; Yang, Y.; Wu, B.; Cheng, G.; Lai, P. B.; Wong, N.; Sung, J. J. Y.; Yu, J., Squalene epoxidase drives NAFLD-induced hepatocellular carcinoma and is a pharmaceutical target. Sci Transl Med 2018,10 (437).

26. Xiao, Z.; Shen, J.; Zhang, L.; Li, M.; Hu, W.; Cho, C., Therapeutic targeting of noncoding RNAs in hepatocellular carcinoma: Recent progress and future prospects. Oncol Lett 2018,15 (3), 3395-3402. 
27. Sassen, S.; Miska, E. A.; Caldas, C., MicroRNA: implications for cancer. Virchows Arch 2008,452 (1), 110.

28. Qiu, L.; Tang, Q.; Li, G.; Chen, K., Long non-coding RNAs as biomarkers and therapeutic targets: Recent insights into hepatocellular carcinoma. Life Sciences 2017, S0024320517305118.

29. Zheng, C.; Liu, X.; Chen, L.; Xu, Z.; Shao, J., IncRNAs as prognostic molecular biomarkers in hepatocellular carcinoma: A systematic review and meta-analysis. Oncotarget 2015,8 (35), 5963859647.

30. Zhao, F.; Qu, Y.; Liu, J.; Liu, H.; Zhang, L.; Feng, Y.; Wang, H.; Gan, J.; Lu, R.; Mu, D., Microarray Profiling and Co-Expression Network Analysis of LncRNAs and mRNAs in Neonatal Rats Following Hypoxicischemic Brain Damage. Sci Rep 2015,5, 13850.

31. Zhou, J.; Lin, J.; Zhang, H.; Zhu, F.; Xie, R., LncRNA HAND2-AS1 sponging miR-1275 suppresses colorectal cancer progression by upregulating KLF14. Biochem Biophys Res Commun 2018,503 (3), 1848-1853.

32. Xu, C.; Ping, Y.; Zhao, H.; Ning, S.; Xia, P.; Wang, W.; Wan, L.; Li, J.; Zhang, L.; Yu, L.; Xiao, Y., LncNetP, a systematical IncRNA prioritization approach based on ceRNA and disease phenotype association assumptions. Oncotarget 2017,8 (70), 114603-114612.

33. Charostad, J.; Astani, A.; Goudarzi, H.; Faghihloo, E., DNA methyltransferases in virus-associated cancers. Rev Med Virol 2019,29 (2), e2022.

34. Jing, W.; Song, N.; Liu, Y.; Qu, X.; Hou, K.; Yang, X.; Che, X., DNA methyltransferase 3a modulates chemosensitivity to gemcitabine and oxaliplatin via CHK1 and AKT in p53deficient pancreatic cancer cells. Mol Med Rep 2018,17(1), 117-124.

35. Wei, D.; Yu, G.; Zhao, Y., MicroRNA-30a-3p inhibits the progression of lung cancer via the PI3K/AKT by targeting DNA methyltransferase 3a. Onco Targets Ther 2019,12, 7015-7024.

36. Wang, L.; Yao, J.; Sun, H.; He, K.; Tong, D.; Song, T.; Huang, C., MicroRNA?101 suppresses progression of lung cancer through the PTEN/AKT signaling pathway by targeting DNA methyltransferase???3A. Oncology Letters 2016.

\section{Figures}


A

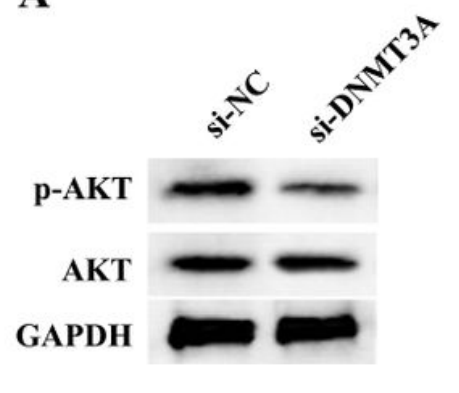

B
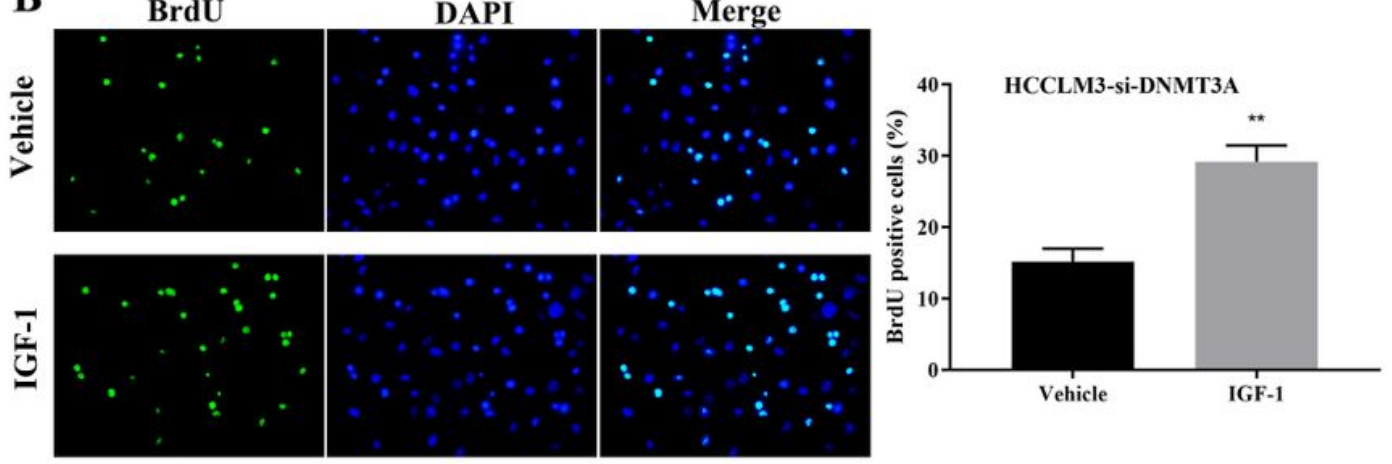

D HCCLM3-si-DNMT3A
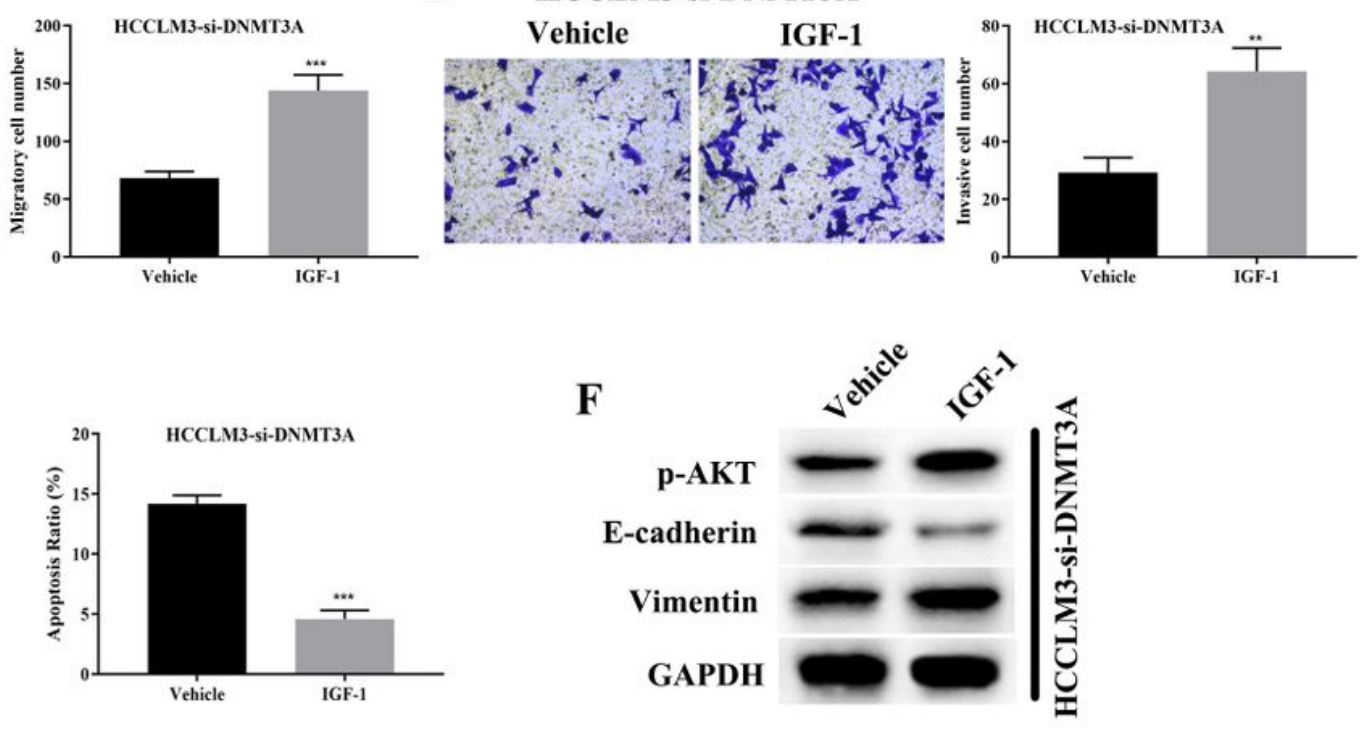

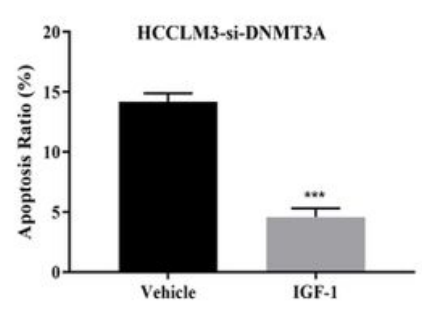

$\sum_{\substack{1 \\ .1}}^{.1}$
E HCCLM3-si-DNMT3A

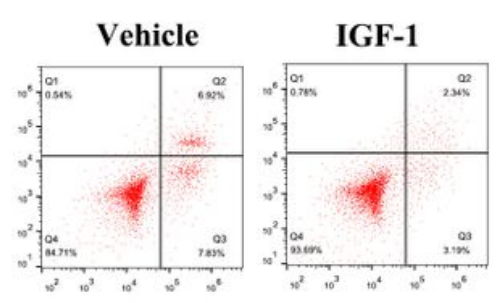

\section{Figure 1}

AKT participated in regulating HCC proliferation, migration and invasion through mediating cell apoptosis and EMT progress. (a) Knockdown of DNMT3A decreased the phosphorylation of AKT. (b) Cell proliferation was augmented when given AKT activator IGF-1 ( $* * p<0.01)$. (c-d) IGF-1 reversed cell migration and invasion that decreased by down-regulation of DNMT3A (**p<0.01, $\left.{ }^{* \star *} p<0.001\right)$. (e-f) IGF-1 prevented cell apoptosis and activated EMT progress $(\star \star \star p<0.001)$. 
A

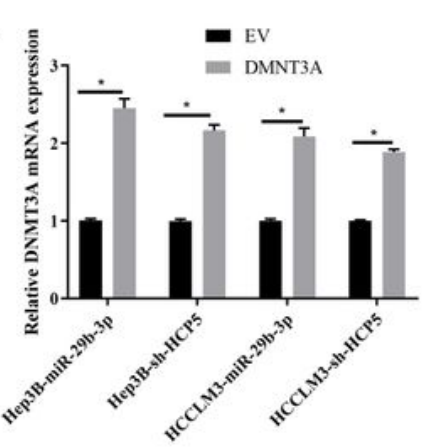

D

(1)

(2)

(3)

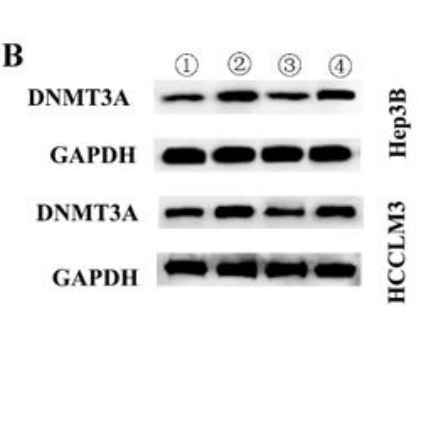

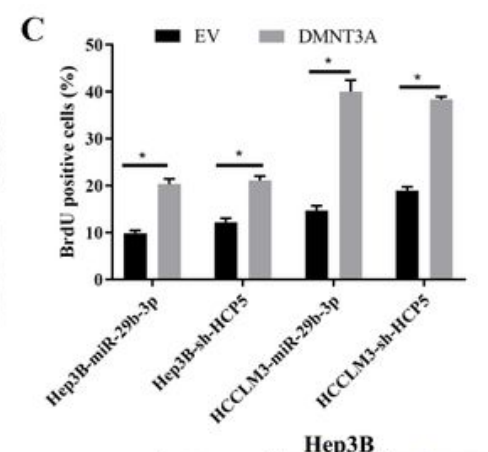
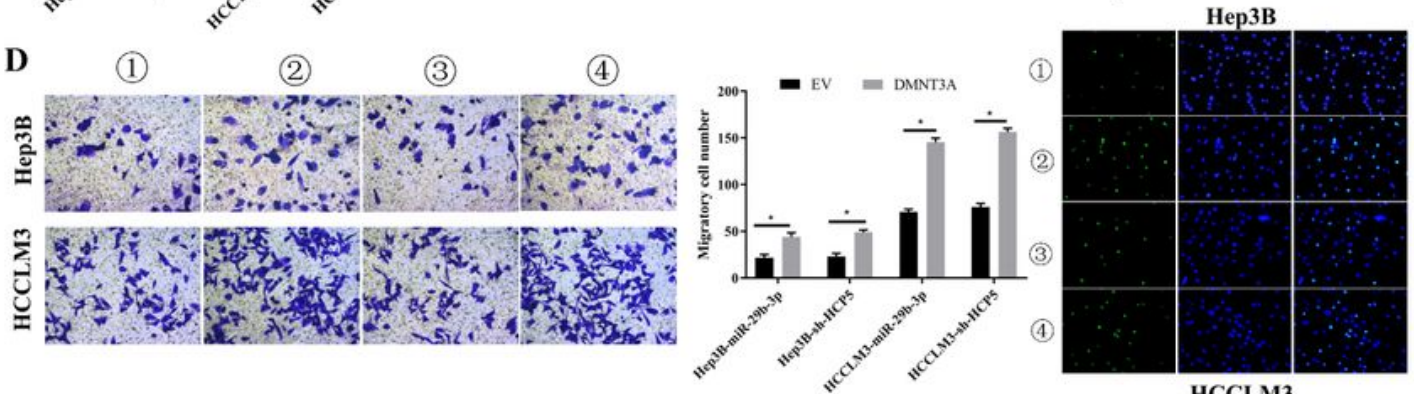

E (1)

(2)

(3)

(4)
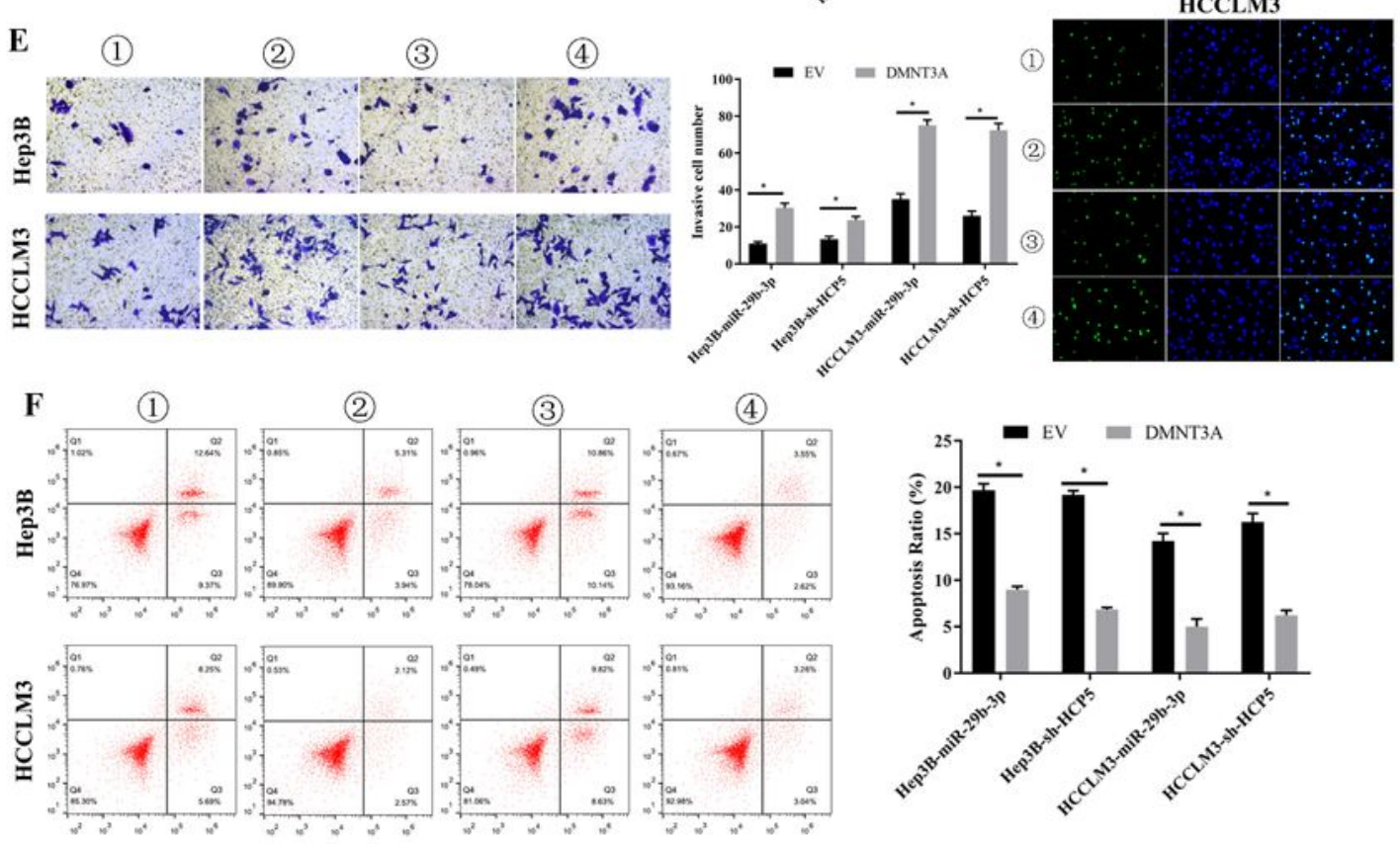

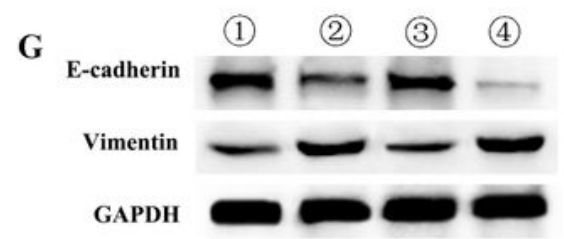

Hep3B
(1) (2) (3) (4)

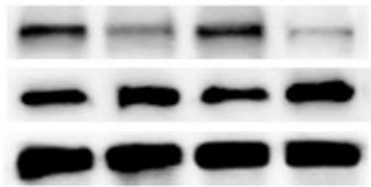

HCCLM3
(1): $\mathbf{m i R}-29 b-3 p+E V$

(2): $\mathbf{m i R}-29 b-3 p+D N M T 3 A$

(3): sh-HCP5+EV

(4):sh-HCP5+ DNMT3A

\section{Figure 2}

DNMT3A promoted HCC proliferation, migration and invasion by activating EMT progress and precluding cell apoptosis. (a-b) The expression of DNMT3A is proportional to HCP5, but is in inverse expression level to miR-29b-3p (*p<0.05). (c) Cell proliferation rate increased when given DNMT3A(*p<0.05). (d-e) DNMT3A promoted cell metastatic and invasive capacity ( $\left.{ }^{*} p<0.05\right)$. (f) DNMT3A promoted proliferation 
through inhibiting cell apoptosis $\left({ }^{\star} \mathrm{p}<0.05\right)$. (g) DNMT3A promoted EMT progress by increasing Ecadherin and decreasing Vimentin.

A

WT DNMT3A 5' CaACCGACUUCAUAAUGGUGCUU 3 '

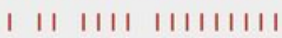

hsa-miR-29b-3p 3' uUguGACUAAAGU-UUACCACGAu 5 ,

C

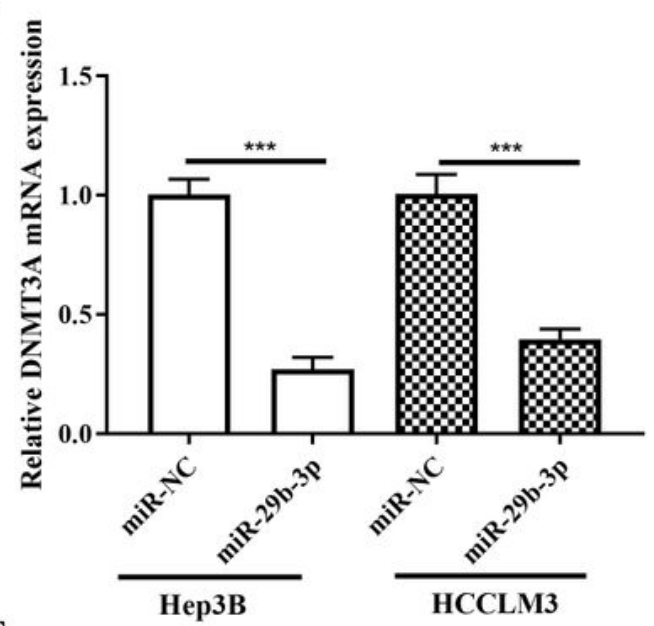

$\mathbf{E}$

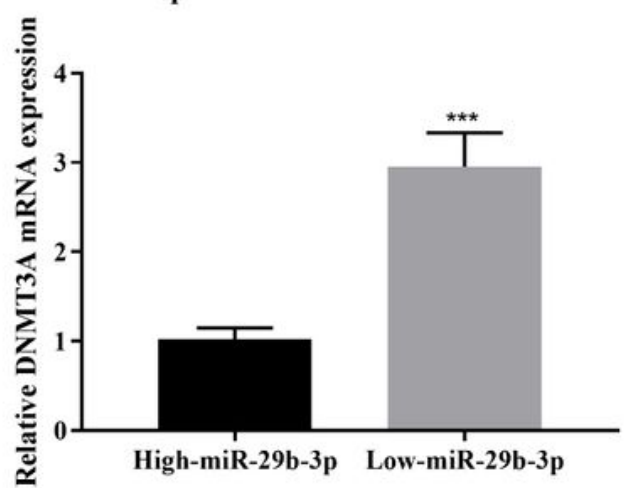

$\mathbf{F}$

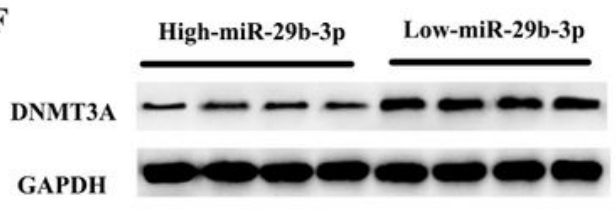

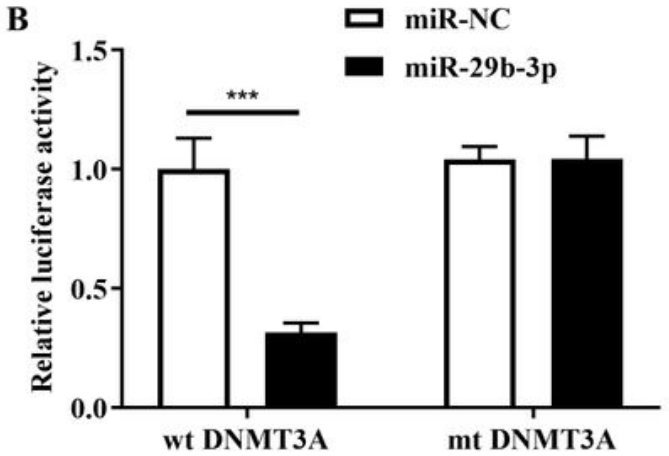

D

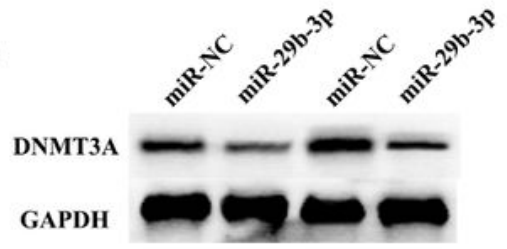

G

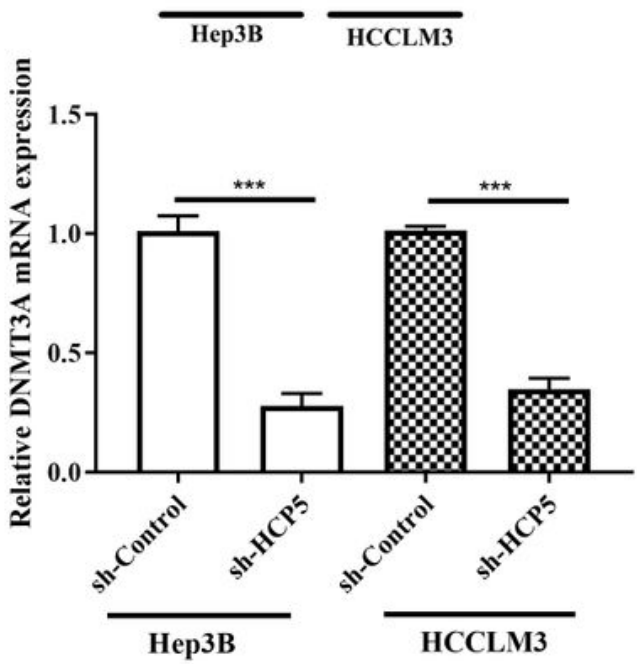

H

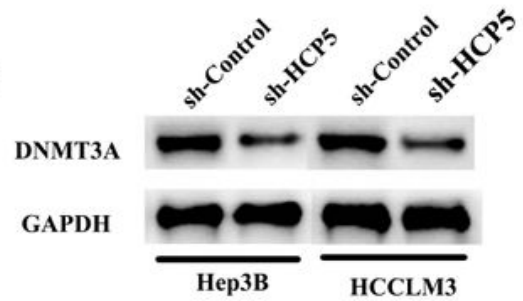

I

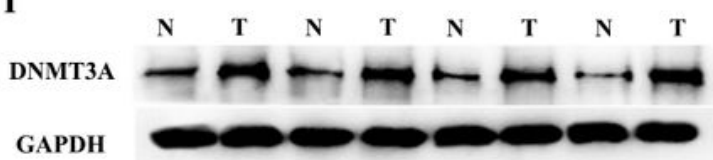

Figure 3

DNMT3A is a negatively regulatory target gene of miR-29b-3p, and DNMT3A promoted HCC proliferation, metastasis and invasion. (a) Bioinformatic prediction showed that there is a direct binding site of miR29b-3p on DNMT3A. (b) Dual luciferase assay demonstrated that there was direct interaction between 
DNMT3A and miR-29b-3p (***p<0.001). (c-e) The results of qRT-PCR and western blot showed that overexpression of miR-29b-3p led to down-regulation of DNMT3A both in HCCLM3 and Hep3B ( $\left.{ }^{* \star} p<0.001\right)$. ( $g$ and $h$ ) qRT-PCR demonstrated that the expression of DNMT3A was proportional to HCP5 $(* \star * p<0.001)$. (f) The expression of DNMT3A was in inverse proportion to miR-29b-3p. (i) Comparation of the expression of DNMT3A in HCC tissues and non-tumor tissues through western blot.
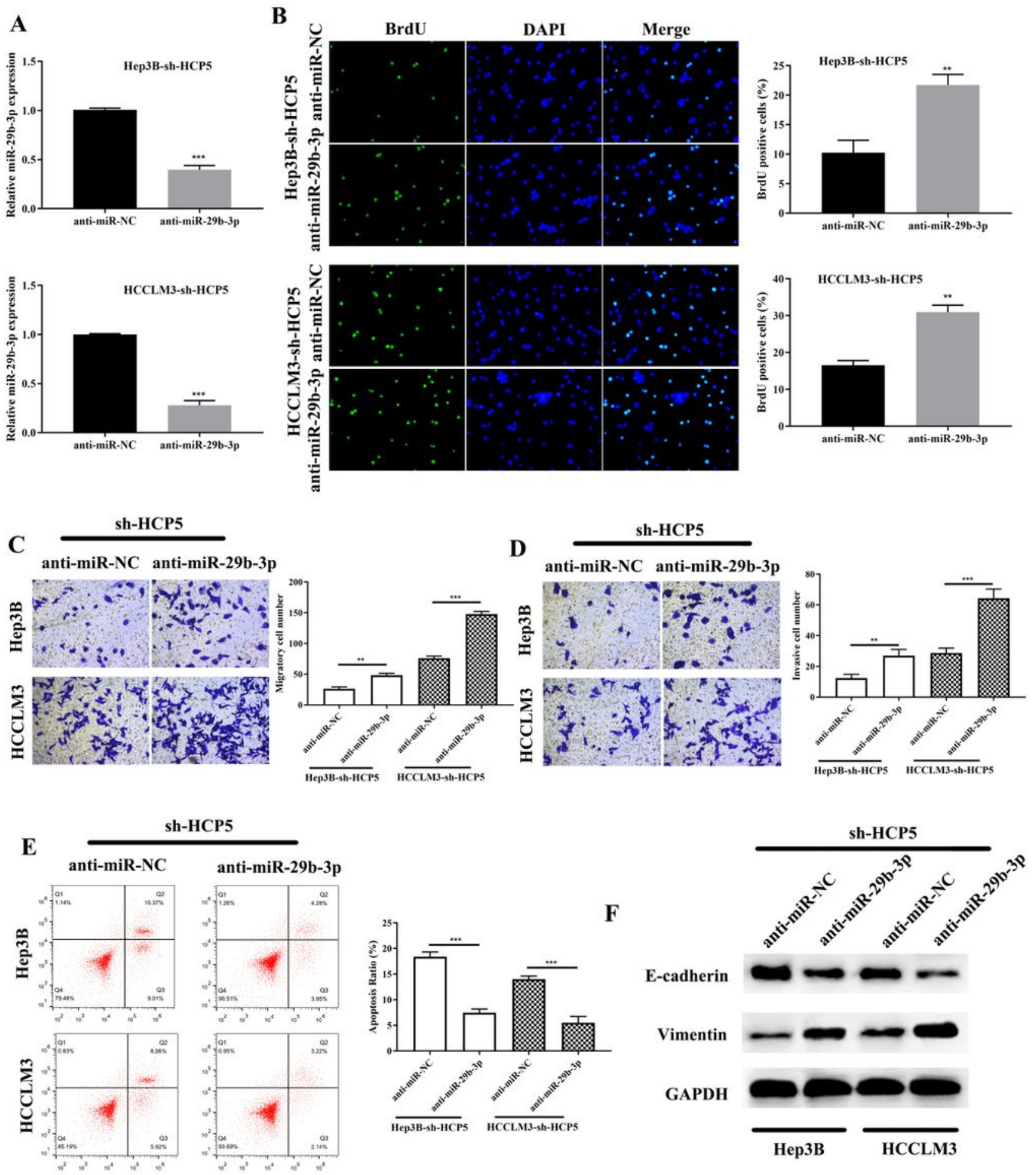

Figure 4 
miR-29b-3p reversed HCC proliferation, migration and invasion that induced by HCP5. (a) The quantities of miR-29b-3p reduced in HCP5 knockdown cells when given anti-miR-29b-3p. (b) miR-29b-3p was upregulated when HCP5 knockdown, while anti- miR-29b-3p was given to cells, the proliferation decreased both in HCCLM3 and Hep3B cell lines ( $\left.{ }^{*} p<0.01\right)$. (c-d) Down-regulation of miR-29b-3p significantly inhibited migration and invasion $(* \star p<0.01, * \star * p<0.001)$. (e) The cell apoptosis decreased when further giving anti-miR-29b-3p to two cell lines with sh-HCP5 transfection. (f) Anti-miR-29b-3p restored the EMT progress induced by HCP5 both in HCCLM3 and Hep3B cell lines.

A
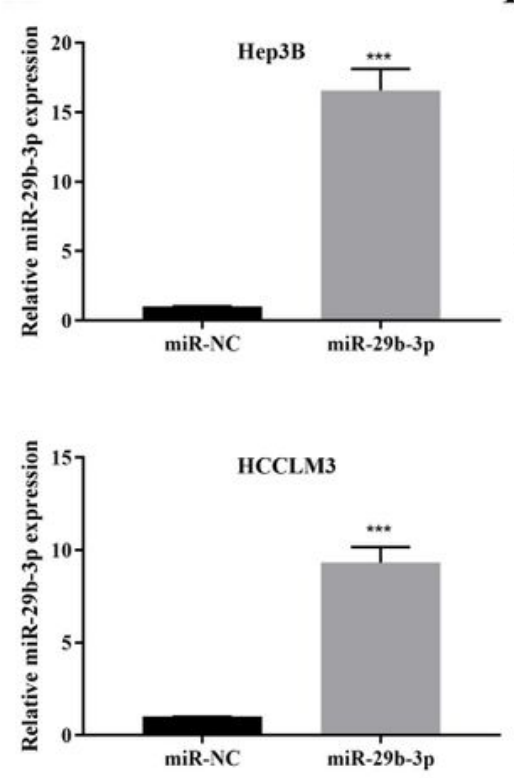

C

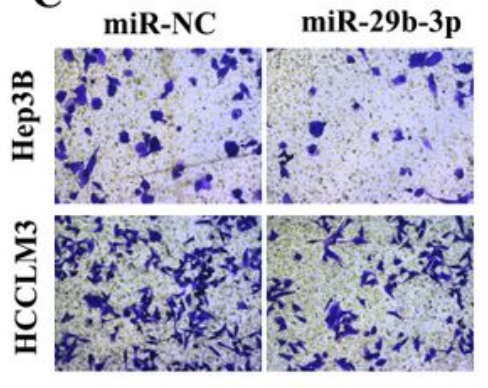

B
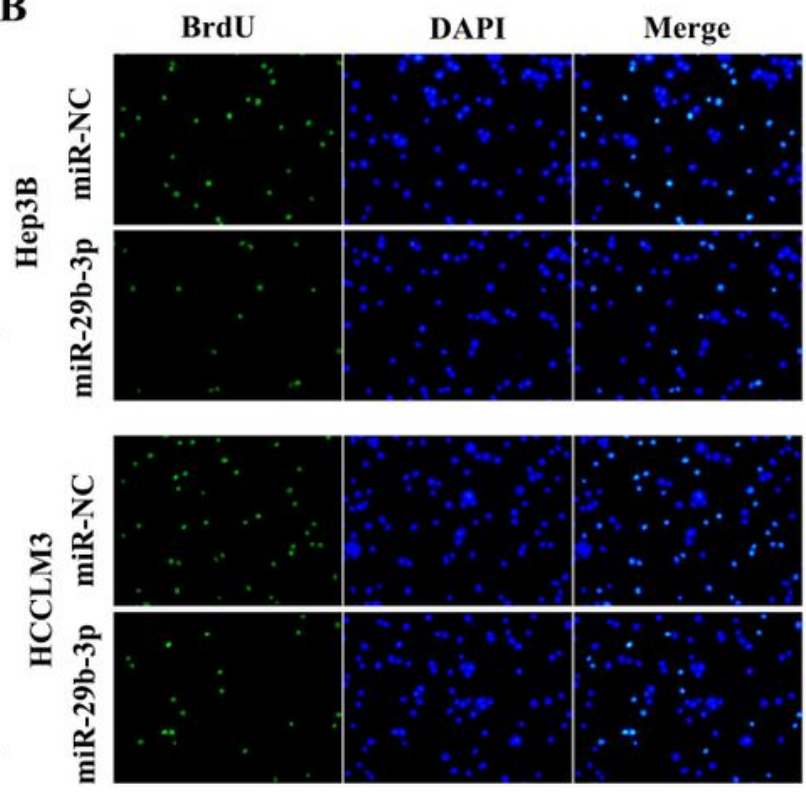
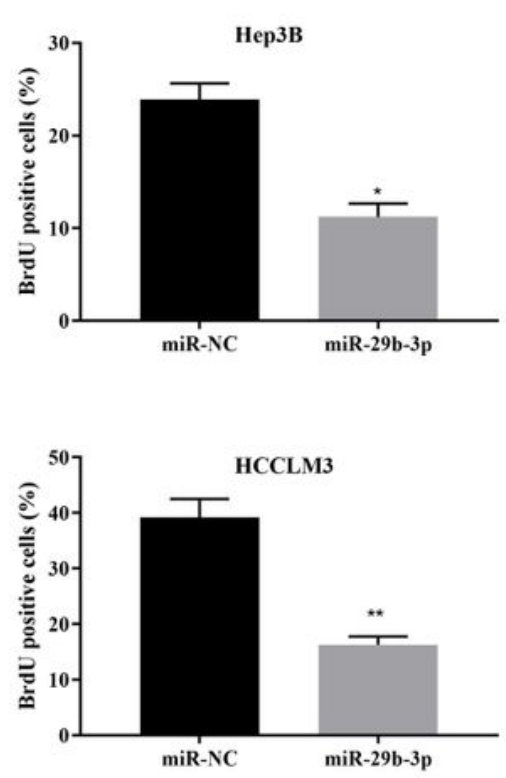

E

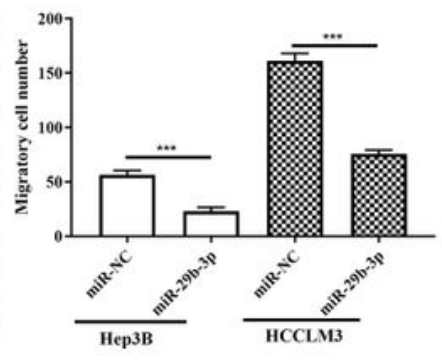

D
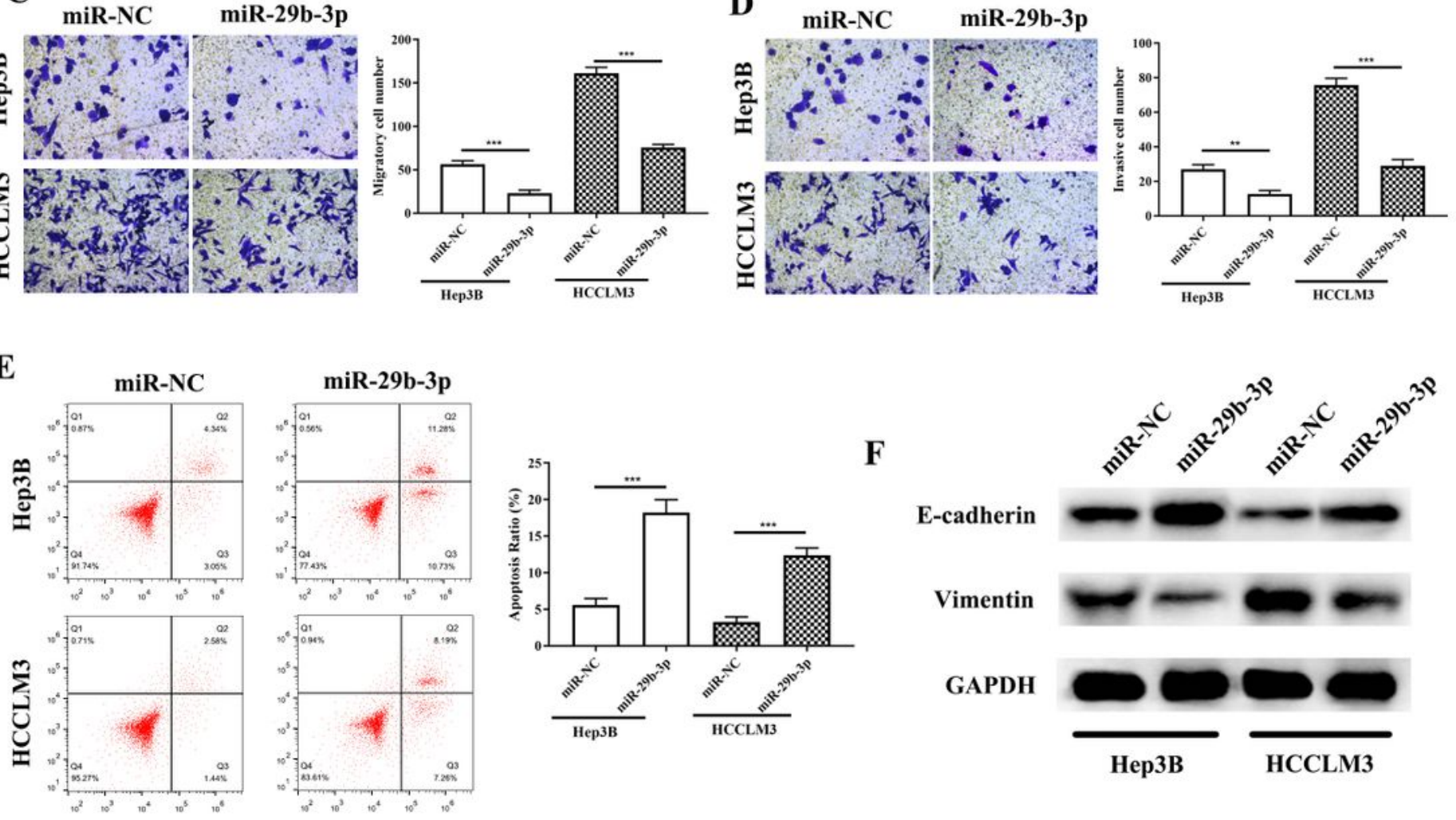

F

E-cadherin

Vimentin

GAPDH

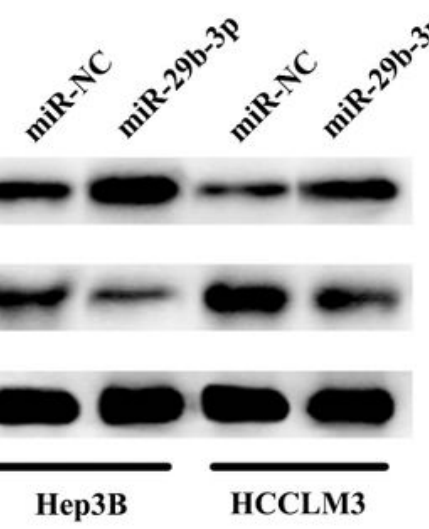




\section{Figure 5}

miR-29b-3p could preclude HCC cell proliferation, migration through enhancing apoptosis and inhibiting EMT progress. (a) After given miR-29b-3p mimic, HCCLM3 and Hep3B cell lines expressed high quantities of miR-29b-3p when compared to miR-control. (b) Cells with high quantities level of miR-29b-3p demonstrated lower proliferation rate $\left({ }^{\star} p<0.05,{ }^{* \star} p<0.01\right)$. (c-d) miR-29b-3p significantly reduced HCC cell migration and invasion ( $\left.{ }^{\star} p<0.01, \star \star \star p<0.001\right)$. (e) Cell apoptosis was improved by miR-29b-3p $(\star \star \star p<0.001)$. (f) Western blot showed that overexpression of miR-29b-3p resulted in up-regulation of Ecadherin and descent quantities of Vimentin. 
A

WT HCP5 5' ucCACUGUGACUCUCCUACUGGUGCUu 3 '

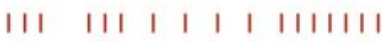

hsa-miR-29b-3p 3' uUGUG--ACU-A-AAGUUUACCACGAu 5 '

B

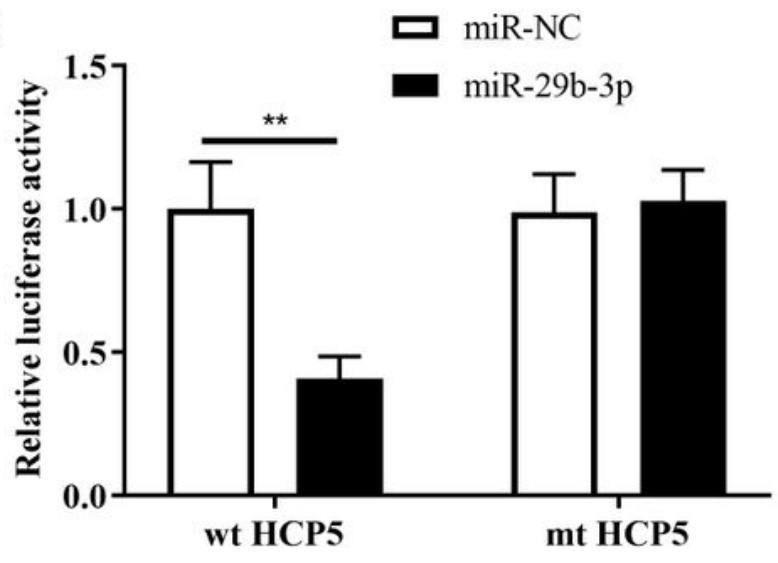

D

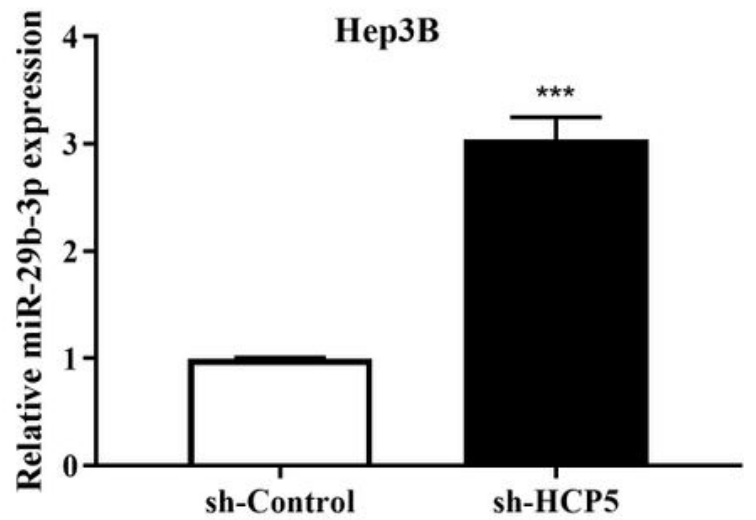

$\mathbf{E}$

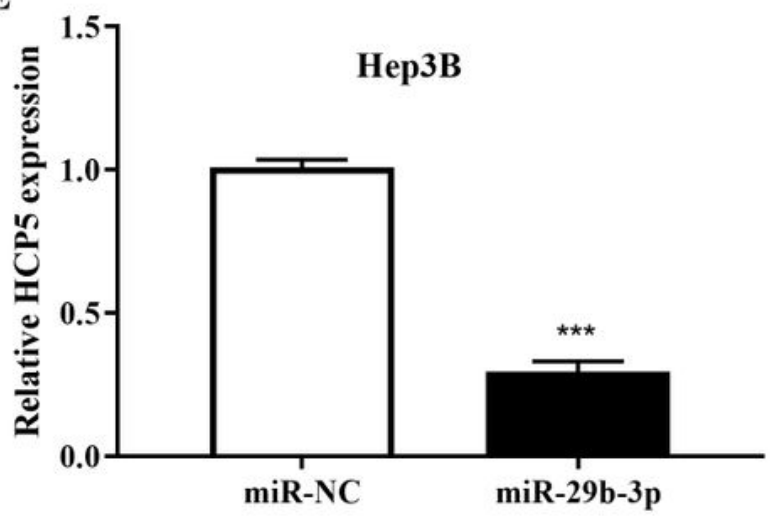

C
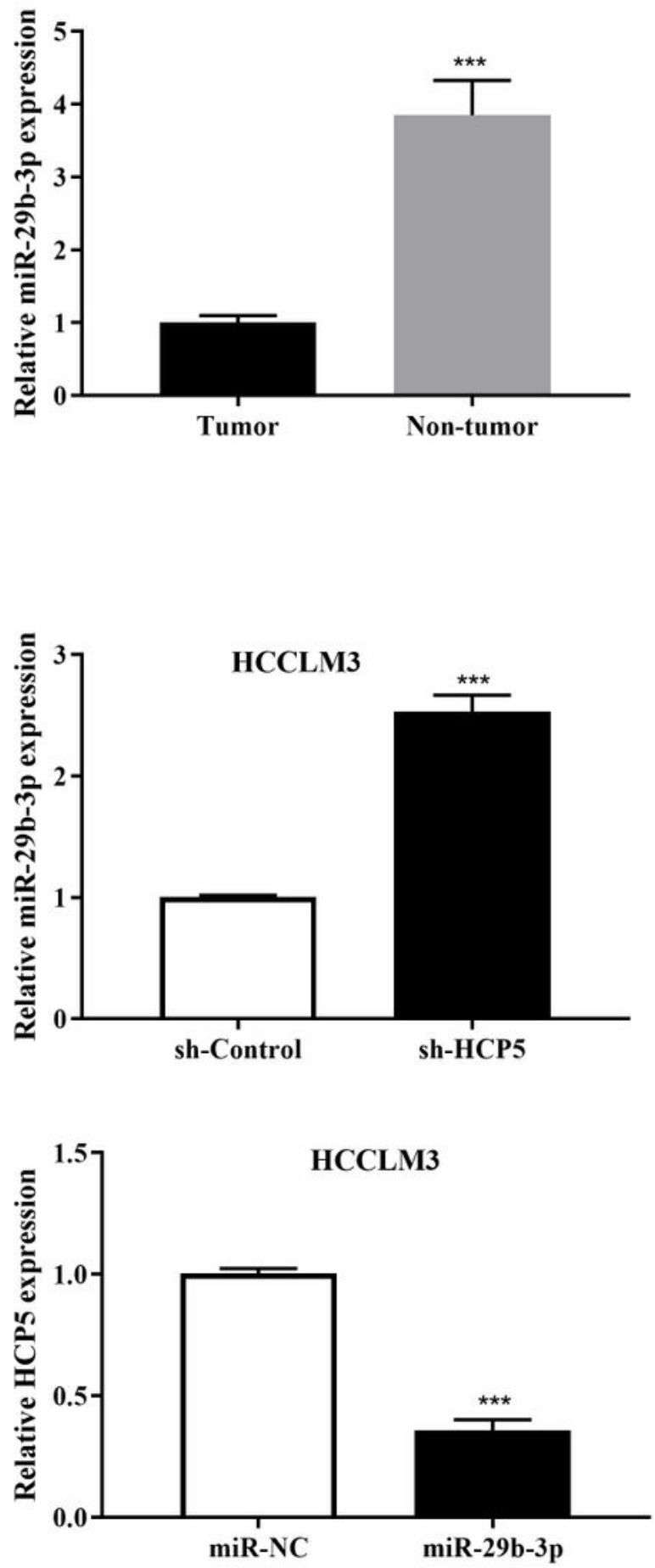

\section{Figure 6}

MicroRNA miR-29b-3p was a negatively regulatory target gene of IncRNA HCP5. (a) Bioinformatic prediction demonstrated that a direct HCP5 binding site existed in miR-29b-3p. (b) Dual luciferase assay demonstrated that miR-29b-3p and HCP5 had direct interaction ( $\star^{*} p<0.01$ ). (c) MiR-29b-3p was in lowerthan-usual expression quantities in HCC tumor tissues when compared to non-tumor tissues $\left({ }^{* *} \mathrm{p}<0.001\right)$. (d) The results of qRT-PCR showed that knockdown of HCP5 inhibited the expression of 
miR-29b-3p both in HCCLM3 and Hep3B cell lines ( $\left.{ }^{* \star} p<0.001\right)$. (e) The results of qRT-PCR showed that knockdown of miR-29b-3p prohibited the expression of HCP5 $(* \star \star p<0.001)$.

A
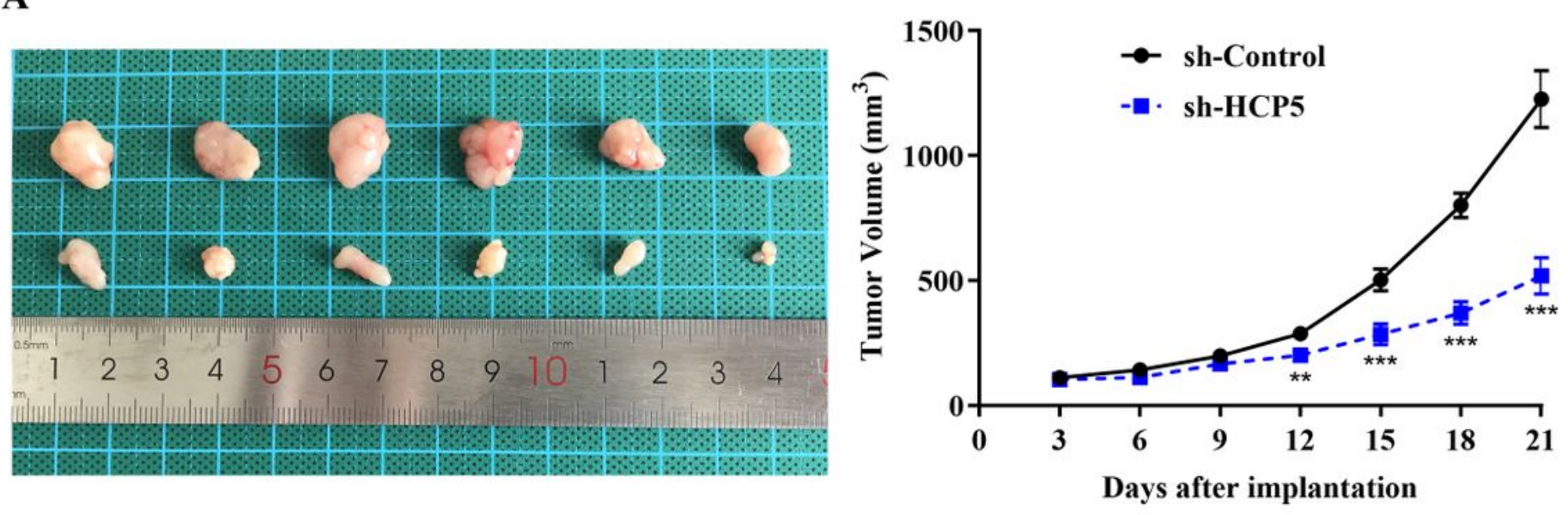

\section{B \\ HE}

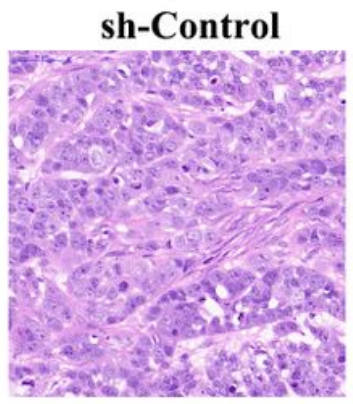

D

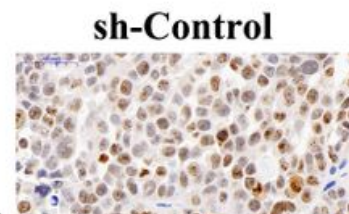

Ki67

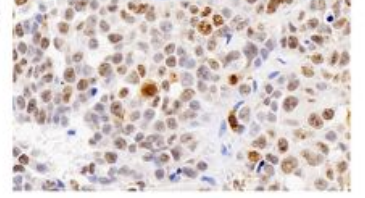

sh-HCP5
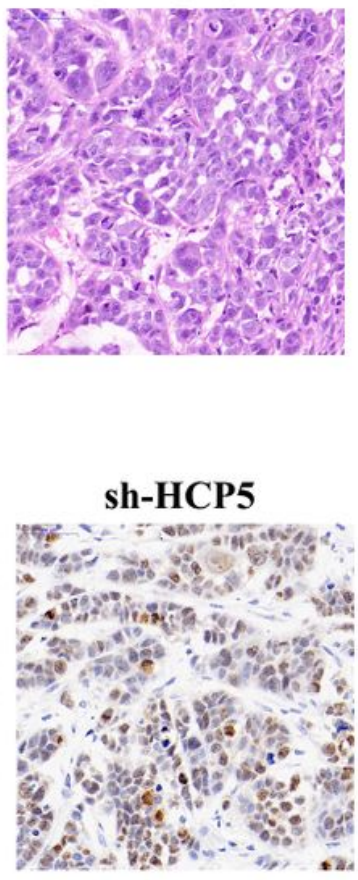

E-cadherin

C

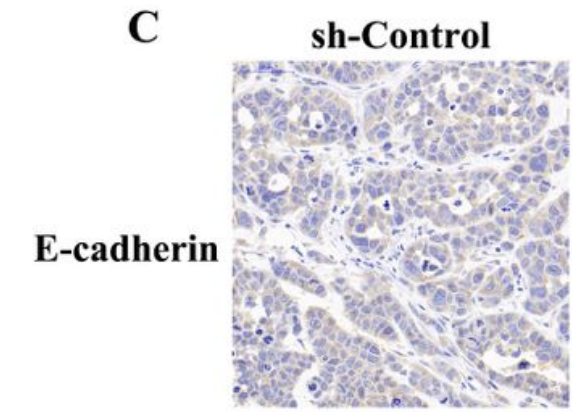

Vimentin
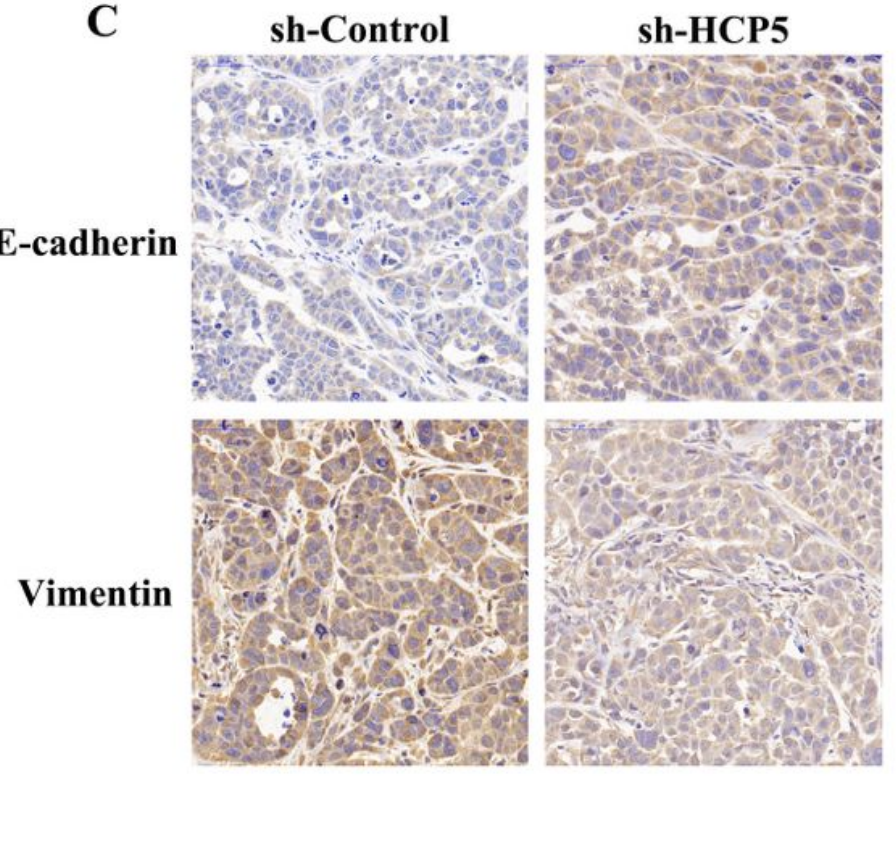

\section{Figure 7}

LncRNA HCP5 promotes HCC growth and migration in vivo. (a) Tumor growth curve suggested that knockdown of HCP5 resulted in lower tumor growth in vivo ( ${ }^{\star *} p<0.01$, $\left.{ }^{\star \star *} p<0.001\right)$. (b) Results of HE staining. (c) Immunohistochemistry demonstrated the expression quantities of E-cadherin and Vimentin between tissues with sh-control and sh-HCP5 transfection. (d) Immunohistochemistry illustrated that knockdown of HCP5 resulted in lower proliferation rate in HCC tissue when compared to control group. 

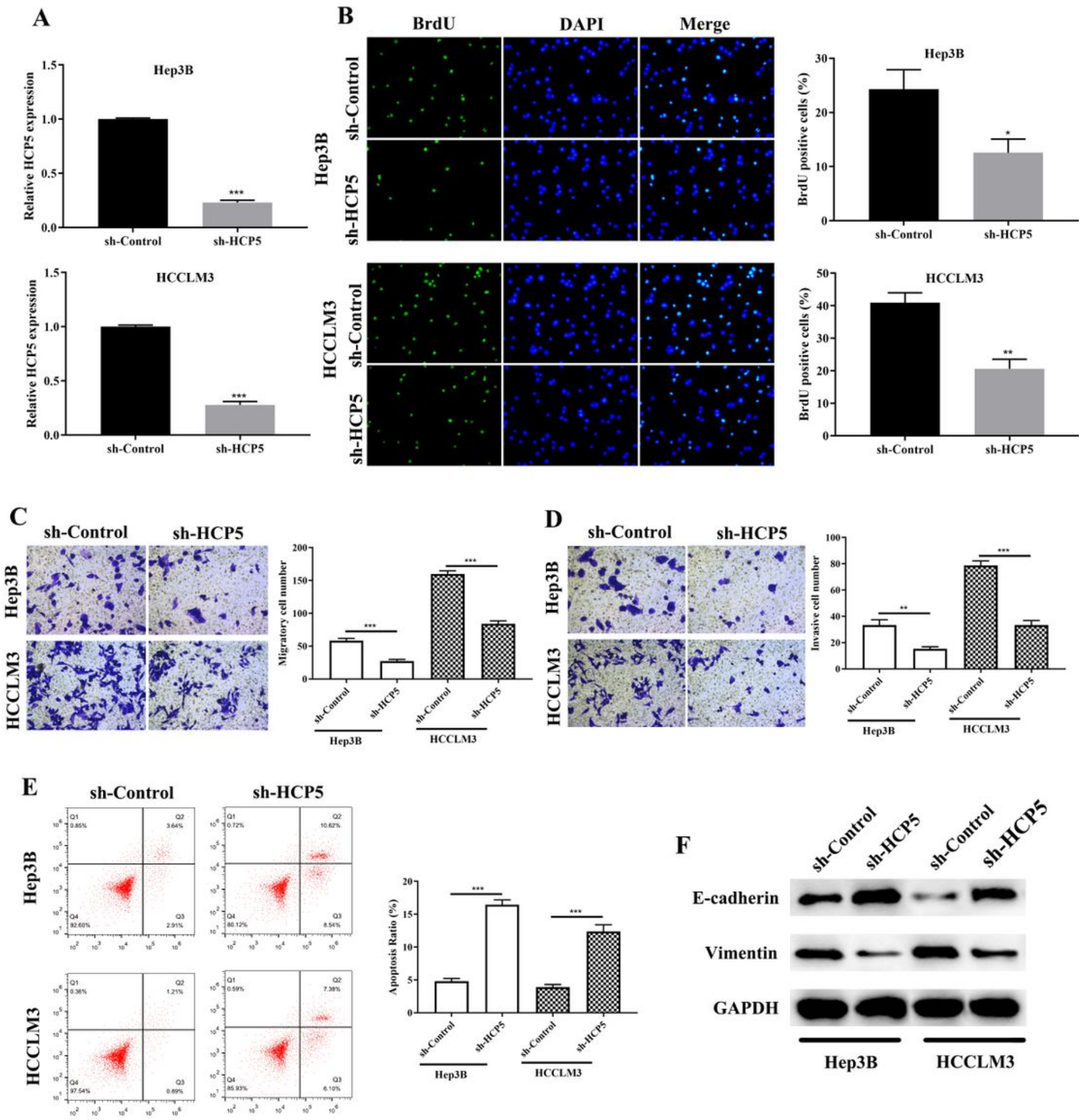

$\mathbf{F}$

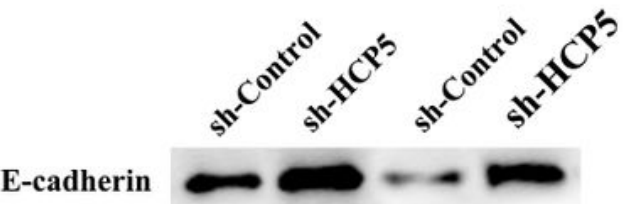

Vimentin

GAPDH

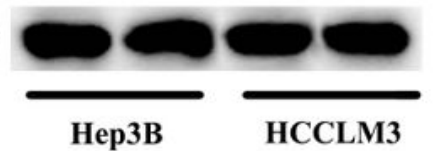

\section{Figure 8}

LncRNA HCP5 augments cell growth, metastasis, invasion, EMT process and prohibits HCC cell apoptosis. (a) Construction of HCP5 knockdown in Hep3B and HCCLM3 cell lines were successful using sh-HCP5 (***p<0.001). (b) Compared to sh-control, knockdown of HCP5 in HCCLM3 and Hep3B prohibited cell proliferation ( $\left.{ }^{*} p<0.05, * *<0.01\right)$. (c-d) Down-regulation of HCP5 decreased migration and invasion in HCCLM3 and Hep3B cell lines ( $\left.{ }^{* *} p<0.001,{ }^{* *} p<0.01\right)$. (e) Cell apoptosis was promoted in 
HCP5 knockdown cells (***p<0.001). (f) Western blot showed that EMT related proteins E-cadherin was overexpressed in HCP5 down-regulation group while the expression of Vimentin was inhibited.

A
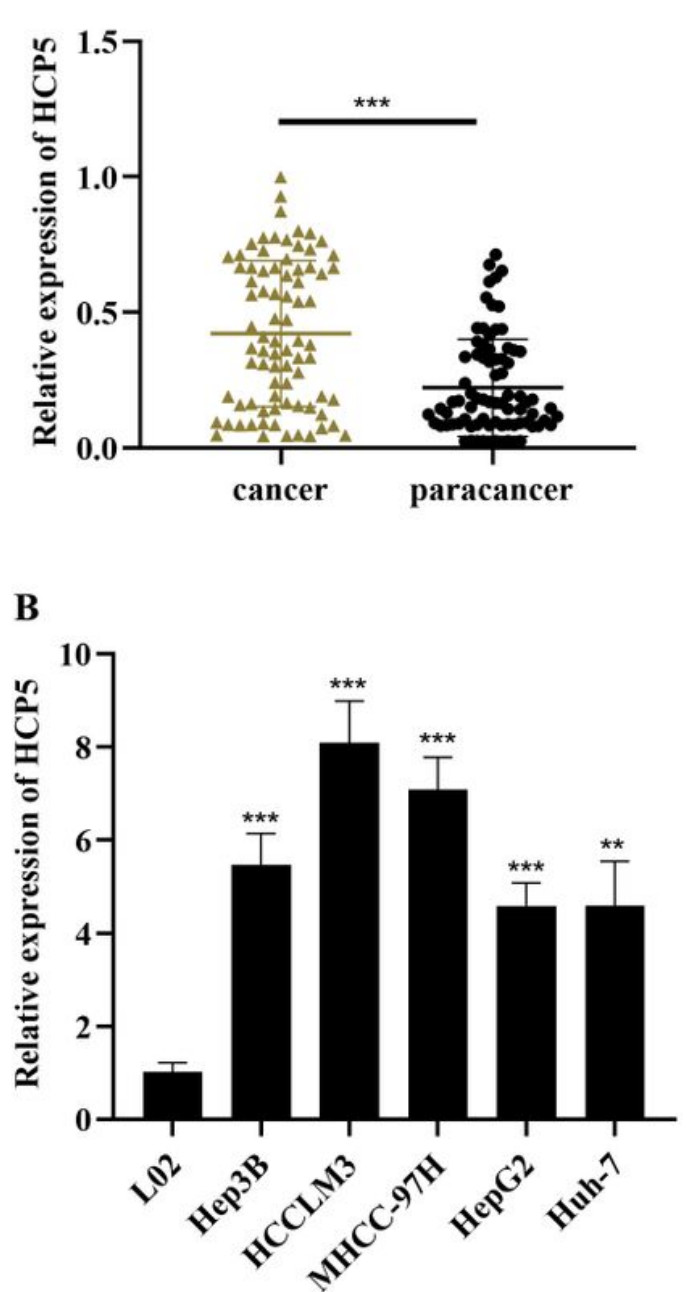

C
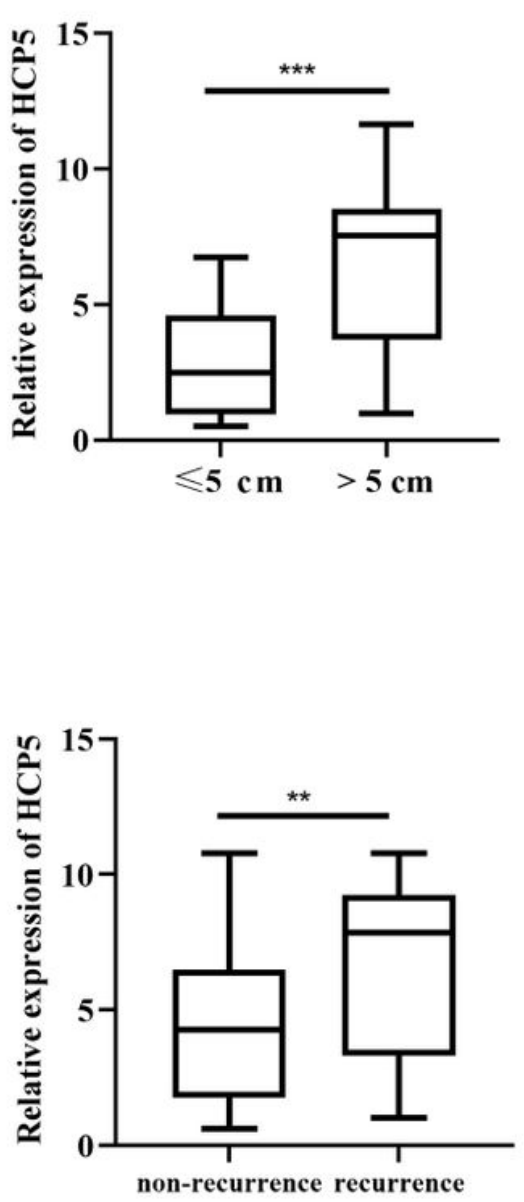
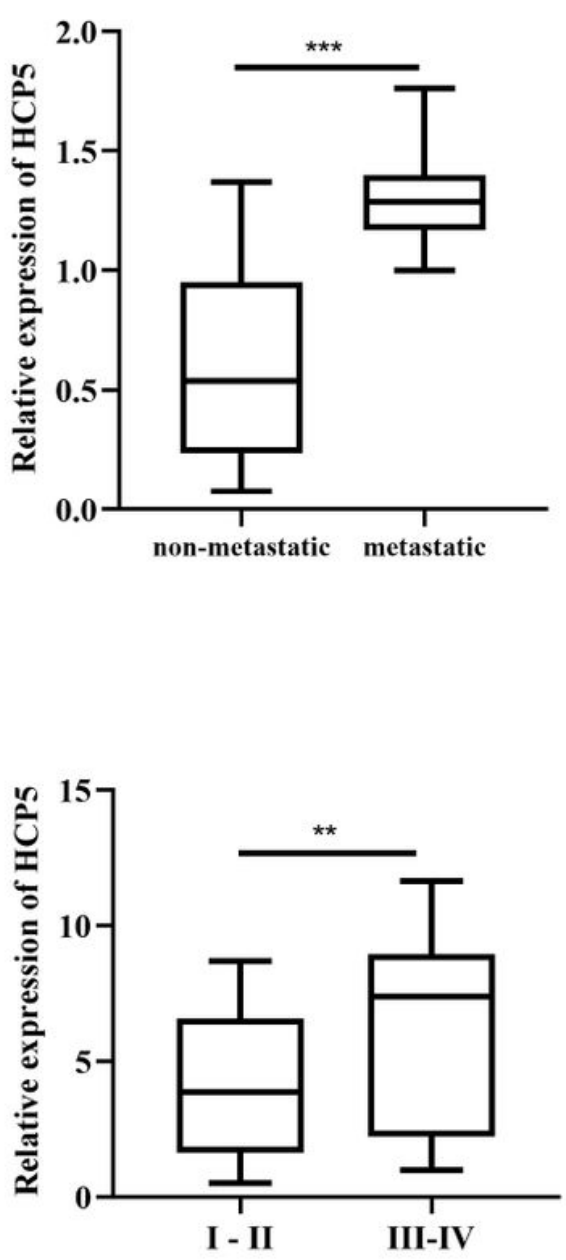

Figure 9

LncRNA is overexpressed in HCC tissues and correlated cell lines. (a) The expression level of HCP5 in HCC tissues is significantly higher than paracancer tissues ( $* \star * p<0.001)$. (b) HCP5 is overexpressed in HCC cell lines ( $\left.{ }^{* *} p<0.001,{ }^{*} p<0.01\right)$, and is in low expression level in normal hepatocytes. (c) The expression of HCP5 in tumor size ( $>5 \mathrm{~cm})$, migrated HCC tissues, recurrent cancer tissues, and high

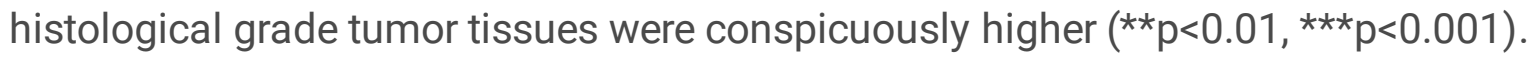

